Research Article

\title{
Monotonicity Preserving Rational Quadratic Fractal Interpolation Functions
}

\author{
A. K. B. Chand and N. Vijender \\ Department of Mathematics, Indian Institute of Technology Madras, Chennai 600036, India \\ Correspondence should be addressed to A. K. B. Chand; chand@iitm.ac.in
}

Received 4 June 2013; Accepted 13 October 2013; Published 30 January 2014

Academic Editor: Hassan Safouhi

Copyright (c) 2014 A. K. B. Chand and N. Vijender. This is an open access article distributed under the Creative Commons Attribution License, which permits unrestricted use, distribution, and reproduction in any medium, provided the original work is properly cited.

\begin{abstract}
Fractal interpolation is an advanced technique for analysis and synthesis of scientific and engineering data. We introduce the $\mathscr{C}^{1}$-rational quadratic fractal interpolation functions (FIFs) through a suitable rational quadratic iterated function system (IFS). The novel notion of shape preserving fractal interpolation without any shape parameter is introduced through the rational fractal interpolation model in the literature for the first time. For a prescribed set of monotonic data, we derive the sufficient conditions by restricting the scaling factors for shape preserving $\mathscr{C}^{1}$-rational quadratic FIFs. A local modification pertaining to any subinterval is possible in this model if the scaling factors are chosen appropriately. We establish the convergence results of a monotonic rational quadratic FIF to the original function in $\mathscr{C}^{4}$. For given data with derivatives at grids, our approach generates several monotonicity preserving rational quadratic FIFs, whereas this flexibility is not available in the classical approach. Finally, numerical experiments support the importance of the developed rational quadratic IFS scheme through construction of visually pleasing monotonic rational fractal curves including the classical one.
\end{abstract}

\section{Introduction}

The interpolation of smooth curve shape constitutes a major research area for reconstruction and representation problems in medical imaging, computer aided geometric design, robotics, automobile engineering, architecture, and multimedia data representation. In manufacturing science, mathematical models to relate part characteristics with process parameters are typically developed from experimental data where the physics based models are not available. The fractal interpolation is an advance technique in fitting of nonsmooth and smooth data from a physical or experimental set-up. To approximate data that follows some kind of self-similarity under magnification, Barnsley [1] introduced fractal interpolation functions (FIFs) defined on a compact interval in $\mathbb{R}$ based on the concept of an IFS [2]. These FIFs are not necessarily differentiable, and they differ from classical interpolants in the sense that (i) FIFs obey an implicit functional relation and (ii) FIFs have noninteger fractal dimensions in general. Since FIFs are being able to extrapolate patterns from one scale to all scales, the use of these functions divulge the presence of an underlying determinism in apparently disorganized data. FIFs have been extensively used due to their characterization for either the generation of geometrically complex graphs of continuous functions or fitting of experimental data. The fractal dimension of a FIF is used to measure the complexity of a signal, and in this way it allows an automatic comparison of recordings [3]. The power of fractal methodology enables the generalization almost any other interpolation techniques; see, for instance, $[4,5]$. The study of spline FIFs has been initiated by Barnsley and Harrington [6], wherein the construction is based on an algebraic method. Due to restricted boundary conditions in this construction, fractal splines with general boundary conditions have been studied recently [7-11]. The studies of IFSs have provided powerful tools for the investigation of fractal sets that are used for approximation of natural or scientific data. The determination of an IFS approximating prescribed data is called "the inverse problem" in the FIF theory. Few works have been reported on this subject based on the FIF model. Strahle [12] found a method to determine 
the scaling parameters in the description of FIFs for such an inverse problem. A different approach in determining the IFS parameters is proposed by Mazel and Hayes [13]. LevkovichMaslyuk [14] and Berkner [15] used wavelet analysis to determine the IFS parameters for the reconstruction of a prescribed curve. Guérin et al. [16] proposed the projected IFS model to approximate rough curves. The shape preserving capabilities of FIFs are not explored in the literature due to their implicit representations. In the CAGD application, particularly in reverse engineering, where the shape is reconstructed from optical scanned data, any curve/surface design should confirm to the overall shape as described by the data. Fractal interpolation can help in preserving local variation but still confirming to the global shape as described by the data. Some typical examples are modelling biological shapes for computational modelling and analysis of biomedical design and haptic surgical simulation or developing virtual models of architectural monuments for digital preservation. In order to construct the shape preserving fractal interpolants in these areas, the paper initiates the theory of monotonicity preserving smooth curves through the rational quadratic FIF models.

After Schoenberg [17, 18] introduced "spline functions" to the mathematical literature, splines have proved to be enormously important in smooth curve representations to discrete data. For smooth curve interpolants, it is crucial to incorporate the inherited features of given data. Data are classified as positive, constrained, monotone, or convex according to their graphs. Schweikert [19] was the first to construct the shape preserving interpolating functions with exponential splines. Shape preserving and cubic spline interpolants with tension parameters were studied in the literature; see, for instance Späth [20], Nielson [21], de Boor [22], and Pruess [23]. Tension parameters were used to control the shape of an interpolant. All these abovementioned methods were $\mathscr{C}^{2}$, global and interpolatory. Automatic algorithms to evaluate the shape parameters by these methods to control shape and monotonicity were involved. In 1980, Fritsch and Carlson [24] introduced a two-pass algorithm for constructing a monotonic piecewise cubic polynomial for a prescribed monotonic data. Fritsch and Butland [25] proposed a modified technique to simplify the Fritsch-Carlson algorithm in 1984. The above two algorithms are both local and produce $\mathscr{C}^{1}$ continuous curves, even if a global $\mathscr{C}^{2}$ solution exists. Furthermore, there is no flexibility in defining the user's desired properties for the resultant spline, for example, the objective function or constraints for an optimization problem. Based on the Fritsch-Carlson algorithm, Costantini proposed several methods to compute shape preserving splines [26-28]. Shape preserving methods based on quadratic spline interpolants have appeared in [29-31] and references therein. The motivation to this work is due to the past work of many authors; for example, the rational quadratic interpolation methodology has been adopted in [32-34]. Rational interpolants play important role in geometric modeling, computer graphics, and CAD/CAM $[35,36]$. For shape preserving interpolants, the rational splines are preferred over ordinary splines [37-39]. Taking the fractal interpolation technique in one hand and the rational interpolation in the other, we introduce the rational FIF in the literature for the first time. In particular, we develop the rational quadratic FIF that preserves desired properties like smoothness and monotonicity as required by a prescribed data set.

In this paper, we initiate the construction of the $\mathscr{C}^{1}$ rational quadratic fractal interpolant through piecewise rational functions whose numerators and denominators are quadratic polynomials. When all scaling factors are zero, in particular, we retrieve the classical piecewise rational quadratic interpolant [32], and this shows the power of generalization of fractal methodology. The rational quadratic FIF described in this paper is unique for a given set of data and a fixed set of scaling factors. The developed rational quadratic fractal scheme is extremely useful when the derivative of the original function is nonsmooth in nature. Monotonicity is an important shape preserving property as uric acid levels in patients suffering from gout, erythrocyte sedimentation rate (ESR) in cancer patients, and data generated from stress of a material are few examples of entities which are always monotonic. This paper examines the shape preserving property monotonicity of the prescribed data through the rational quadratic FIF model. Because of the recursive nature of a FIF, the necessary condition for monotonicity on derivatives at knots alone may not ensure the monotonicity of a rational quadratic fractal interpolant for a given set of monotonic data. Therefore, we derive sufficient conditions for monotonicity based on appropriate conditions on the scaling factors $\alpha_{i}$ such that these conditions together with the necessary conditions ensure the monotonicity of a rational quadratic fractal interpolant for prescribed monotonic data. Our construction does not need any additional points for the rational quadratic FIFs, whereas the quadratic spline methods of Schumaker [30] and the cubic interpolation method of Brodlie and Butt [40] require the introduction of additional points for shape preserving interpolants.

The content of this paper is organized as follows. The mathematical backgrounds of FIFs based on the IFS theory along with the calculus of rational FIFs are discussed in Section 2. In Section 3, the inverse problem of interpolation with a $\mathscr{C}^{1}$-rational quadratic FIF is introduced. We discuss sufficient conditions for these interpolants to be monotonic by deriving appropriate restrictions on the scaling parameters of the associated rational IFS in Section 4, and an upper bound of the uniform error of the monotonic rational quadratic FIF with the original function in $\mathscr{C}^{4}$ is estimated for the convergence results in Section 4.2. Finally, the application of the shape preserving rational quadratic IFS scheme is illustrated on monotonic data set for visually pleasing $\mathscr{C}^{1}$ monotonic rational fractal interpolants, and the effects of change in the scaling parameters on the rational quadratic FIF and its derivative are demonstrated in Section 5.

\section{Fractal Interpolation Functions}

The basics of IFS theory are discussed in Section 2.1, and the construction of a FIF from a suitable IFS is presented 
in Section 2.2. The calculus of the rational FIFs is given in Section 2.3.

2.1. IFS Theory. Suppose $\left(\mathscr{X}, d_{\mathscr{X}}\right)$ is a complete metric space, and $\mathscr{H}(\mathscr{X})=\{A \subset \mathscr{X}: A \neq \phi, A$ is compact $\}$. The Hausdorff metric $h\left(d_{\mathscr{X}}\right)$ on the space $\mathscr{H}(\mathscr{X})$ is defined as $h(A, B)=\max \{d(A, B), d(B, A)\}$, where $d(A, B)=$ $\max _{x \in A} \min _{y \in B} d_{\mathscr{X}}(x, y)$. The space of fractals $\left(\mathscr{H}(\mathscr{X}), h\left(d_{\mathscr{X}}\right)\right)$ is a complete metric space. An IFS $\mathscr{I}=\left\{\mathscr{X} ; w_{i}, i=\right.$ $1,2, \ldots, n-1\}$ is a collection of $n-1$ functions defined on the complete metric space $\left(\mathscr{X}, d_{\mathscr{X}}\right) . \mathscr{I}$ is called a hyperbolic IFS if $w_{i}$ is a contraction map (say) with contractive factor $\left|s_{i}\right|<1$ for $i=1,2, \ldots, n-1$. The Hutchinson map [2] on $\mathscr{H}(\mathscr{X})$ is defined as $W(A)=\cup_{i=1}^{n-1} w_{i}(A)$ for all $A \in \mathscr{H}(\mathscr{X})$. Now, $W$ is a contraction map on $\left(\mathscr{H}(\mathscr{X}), h\left(d_{\mathscr{X}}\right)\right)$ with the contractive factor $s=\max \left\{\left|s_{i}\right|: i=1,2, \ldots, n-1\right\}$. By the Banach Fixed Point Theorem, there exists a unique $G \in \mathscr{H}(\mathscr{X})$ such that $\lim _{m \rightarrow \infty} W^{m}(A)=G$ for any $A \in \mathscr{H}(\mathscr{X})$, and this fixed point $G$ is known as an attractor or a deterministic fractal of the hyperbolic IFS. In the inverse problem, $G$ is the object to be approximated by a suitable IFS $\mathscr{I}$. Since an image can be interpreted by its IFS code, the fractal image compression is one of the popular applications in the current research of fractal theory [41]. Based on the IFS theory, a FIF is constructed as the graph of the attractor in the following [1].

2.2. Construction of FIF. Let $x_{1}<x_{2}<\cdots<x_{n-1}<x_{n}$ be a partition of the real compact interval $I=\left[x_{1}, x_{n}\right]$. Let a data set $\left\{\left(x_{i}, f_{i}\right) \in I \times D: i=1,2, \ldots, n\right\}$ be given, where $D$ is compact in $\mathbb{R}$. Set $I_{i}=\left[x_{i}, x_{i+1}\right]$, and let $L_{i}: I \rightarrow I_{i}, i=$ $1,2, \ldots, n-1$, be contractive homeomorphisms such that

$$
\begin{aligned}
& L_{i}\left(x_{1}\right)=x_{i}, \quad L_{i}\left(x_{n}\right)=x_{i+1}, \\
& \left|L_{i}\left(c_{1}\right)-L_{i}\left(c_{2}\right)\right| \leq l_{i}\left|c_{1}-c_{2}\right|, \quad \forall c_{1}, c_{2} \in I,
\end{aligned}
$$

for some $l_{i}<1$. Denote $C=I \times D$. Define the continuous mappings $F_{i}: C \rightarrow D, i=1,2, \ldots, n-1$, such that

$$
\begin{gathered}
F_{i}\left(x_{1}, f_{1}\right)=f_{i}, \quad F_{i}\left(x_{n}, f_{n}\right)=f_{i+1}, \\
\left|F_{i}(x, y)-F_{i}(x, z)\right| \leq\left|\alpha_{i}\right||y-z| \\
x \in I, \quad y, z \in \mathbb{R},-1<\alpha_{i}<1 .
\end{gathered}
$$

For the construction of a desired IFS, now define the functions $w_{i}: C \rightarrow I_{i} \times D(i=1,2, \ldots, n-1)$ as $w_{i}(x, f)=$ $\left(L_{i}(x), F_{i}(x, f)\right)$. The construction of a FIF is based on the following results.

Proposition 1 (see [1]). The IFS $\left\{C ; w_{i}, i=1,2, \ldots, n-1\right\}$ defined above admits a unique attractor $G$. $G$ is the graph of a continuous function $h^{*}: I \rightarrow \mathbb{R}$ such that $h^{*}\left(x_{i}\right)=f_{i}$ for $i=1,2, \ldots, n$.

The above function $h^{*}$ is called a FIF corresponding to the IFS $\left\{C ; w_{i}, i=1,2, \ldots, n-1\right\}$, and the construction of $h^{*}$ is based on the following discussion.

Suppose $\mathscr{G}=\left\{g: I \rightarrow \mathbb{R} \mid g\right.$ is continuous, $g\left(x_{1}\right)=$ $f_{1}$, and $\left.g\left(x_{n}\right)=f_{n}\right\}$. Then $\mathscr{G}$ is a complete metric space with respect to the metric $d$ induced by the uniform norm on $\mathscr{C}(I)$. Define the Read-Bajraktarević operator $T$ on $(\mathscr{G}, d)$ by

$$
\begin{array}{r}
\operatorname{Tg}(x)=F_{i}\left(L_{i}^{-1}(x), g\left(L_{i}^{-1}(x)\right)\right), \\
x \in I_{i}, \quad i=1,2, \ldots, n-1 .
\end{array}
$$

According to (1)-(2), $\mathrm{Tg}$ is continuous on the intervals $I_{i}$, $i=1,2, \ldots, n-1$, and at each of the points $x_{2}, \ldots, x_{n-1}$. Also $T$ is a contraction mapping on the metric space $(\mathscr{G}, d)$; that is,

$$
d\left(T g, \operatorname{Tg}^{*}\right) \leq|\alpha|_{\infty} d\left(g, g^{*}\right)
$$

where $|\alpha|_{\infty}=\max \left\{\left|\alpha_{i}\right|: i=1,2, \ldots, n-1\right\}$. Since $|\alpha|_{\infty}<$ 1, T possesses a unique fixed point, say $h^{*}$ on $\mathscr{G}$ such that $\left(T h^{*}\right)(x)=h^{*}(x) \forall x \in I$. According to (3), the FIF satisfies the following functional equation:

$$
\begin{array}{r}
h^{*}(x)=F_{i}\left(L_{i}^{-1}(x), h^{*} \circ L_{i}^{-1}(x)\right), \\
x \in I_{i}, i=1,2, \ldots, n-1 .
\end{array}
$$

The popular IFS to define fractal interpolation functions takes the following form:

$$
L_{i}(x)=a_{i} x+b_{i}, \quad F_{i}(x, f)=\alpha_{i} f+q_{i}(x),
$$

where $-1<\alpha_{i}<1$, and $q_{i}: I \rightarrow \mathbb{R}$ is a suitable continuous function satisfying (2). In FIF theory, $\alpha_{i}$ is called the scaling factor of the transformation $w_{i}$, and $\alpha=\left(\alpha_{1}, \alpha_{2}, \ldots, \alpha_{n-1}\right)$ is the scale vector of the IFS. The scaling factors give additional degrees of freedom to the FIF and allow us to modify its shape and properties. In particular, when $\alpha=\mathbf{0}$, the fixed point $h^{*}(x)$ reduces to some classical piecewise interpolation function, which can be described according to the nature of $q_{i}(x), i=1,2, \ldots, n-1$.

Examples 1. Consider the interpolation data $\{(1,-1),(2,5),(5,-3),(9,8)\}$ with $n=4$. From (1) and (6),

$$
\begin{gathered}
L_{1}(x)=0.125 x+0.875, \quad L_{2}(x)=0.375 x+1.625, \\
L_{3}(x)=0.5 x+4.5 .
\end{gathered}
$$

Suppose $q_{i}(x):=x^{2}+c_{i} x+d_{i}$. The constants $c_{i}$ and $d_{i}$ in $q_{i}$ are calculated using (2) as

$$
\begin{aligned}
& c_{i}=\frac{f_{i+1}-x_{n}^{2}-\alpha_{i} f_{n}-\left(f_{i}-x_{1}^{2}-\alpha_{i} f_{1}\right)}{x_{n}-x_{1}}, \\
& d_{i}=\frac{x_{n}\left(f_{i+1}-x^{2}-\alpha_{i} f_{n}\right)-x_{1}\left(f_{i}-x_{1}^{2}-\alpha_{i} f_{1}\right)}{x_{n}-x_{1}} .
\end{aligned}
$$

From (5), we obtain for $x \in[1,9]$,

$$
h^{*}\left(L_{i}(x)\right)=\alpha_{i} h^{*}(x)+x^{2}+c_{i} x+d_{i}, \quad i=1,2,3 .
$$

$h^{*}$ is called a quadratic FIF. By taking different values of scaling vectors $\alpha=\left(\alpha_{1}, \alpha_{2}, \alpha_{3}\right)$, we can construct smooth or nonsmooth FIFs for given data. For instance, we construct 


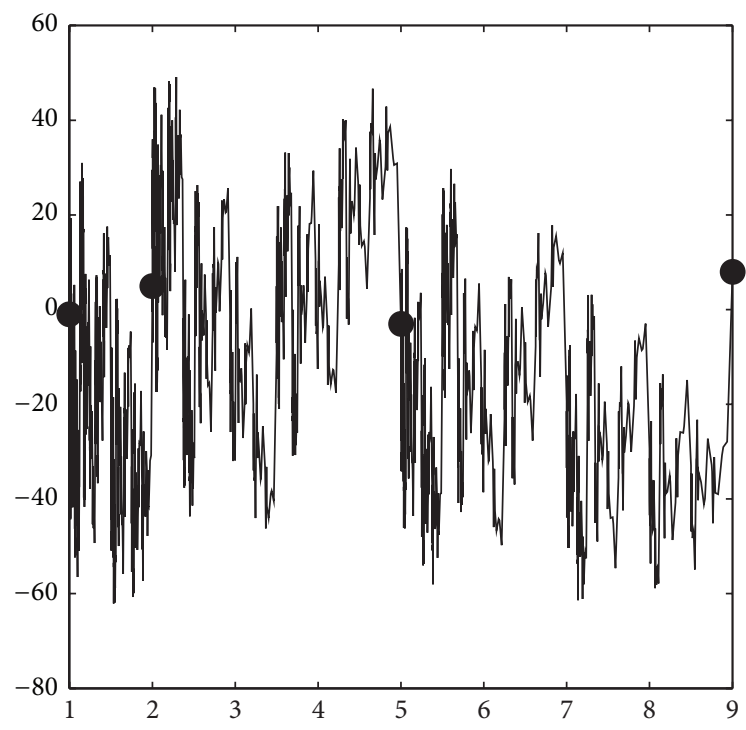

(a) $\operatorname{FIF} h^{*}$ with $\alpha=(0.9,-0.9,0.9)$

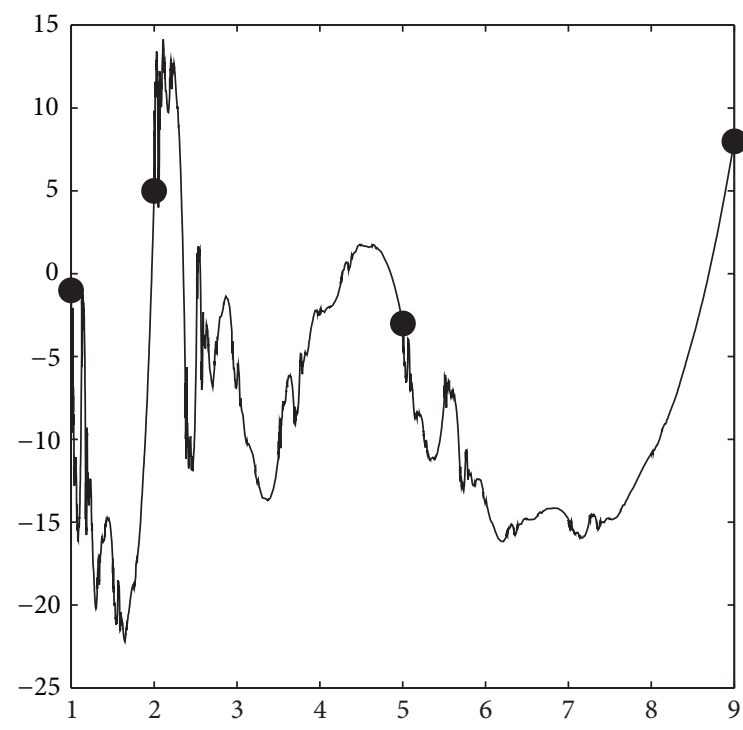

(b) FIF $h^{*}$ with $\alpha=(0.5,-0.6,0.2)$

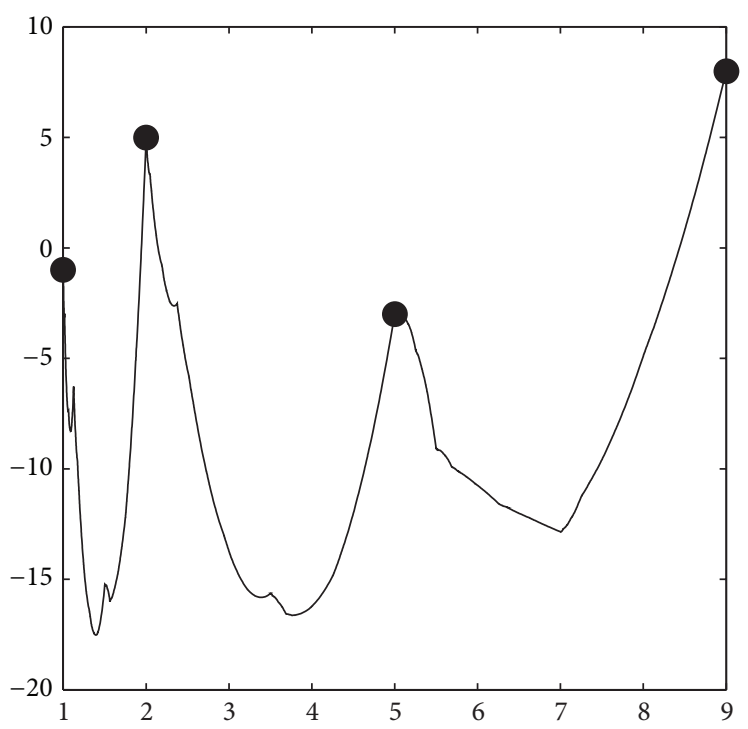

(c) FIF $h^{*}$ with $\alpha=(0.2,0.1,-0.1)$

FIGURE 1: Quadratic FIFs.

Figure 1(a) with $\alpha=(0.9,-0.9,0.9)$, Figure 1(b) with $\alpha=$ $(0.5,-0.6,0.2)$, and Figure $1(\mathrm{c})$ with $\alpha=(0.2,0.1,-0.1)$. It is easy to observe that the smoothness of the quadratic FIF increases in each subinterval as $\left|\alpha_{i}\right| \rightarrow 0$ for all $i$. Now we will discuss the rational FIFs in the following, where $q_{i}(x)$ is taken as a rational function defined on $I$ for $i=$ $1,2, \ldots, n-1$.

2.3. Calculus of Rational FIF. We develop the calculus of the rational FIFs using piecewise rational functions as per theory of a polynomial FIF in [6].
For a rational FIF, we define

$$
\begin{gathered}
L_{i}(x)=a_{i} x+b_{i}, \quad F_{i}(x, f)=\alpha_{i} f+q_{i}(x), \\
q_{i}(x)=\frac{U_{i, M}(x)}{V_{i, N}(x)},
\end{gathered}
$$

where $U_{i, M}$ is a polynomial of degree $M$ and $V_{i, N}$ is a polynomial of degree $N$ such that $V_{i, N}(x) \neq 0$ for every $x \in$ $I$ in (6). Also $q_{i}$ contains two real parameters that can be evaluated by using (2). Let $\Phi$ be a rational FIF related to the 
IFS $\left\{C ; w_{i}(x, f)=\left(L_{i}(x), F_{i}(x, f)\right), i=1,2, \ldots, n-1\right\}$. Let the value of integral of $\Phi$ be known at $x_{1}\left(\right.$ or $\left.x_{n}\right)$ as $\tilde{f}_{1}\left(\right.$ or $\left.\tilde{f}_{n}\right)$. If

$$
\widetilde{\Phi}(x)=\widetilde{f}_{1}+\int_{x_{1}}^{x} \Phi(\tau) d \tau\left(\text { or } \widetilde{\Phi}(x)=\widetilde{f}_{n}-\int_{x}^{x_{n}} \Phi(\tau) d \tau\right)
$$

then $\widetilde{\Phi}$ is a rational fractal function related to the IFS $\{I \times$ $\left.K^{*} ; \widetilde{w}_{i}(x, f)=\left(L_{i}(x), \widetilde{F}_{i}(x, f)\right), i=1,2, \ldots, n-1\right\}$, where $K^{*}$ is a suitable compact subset of $\mathbb{R}$,

$$
\begin{gathered}
\tilde{F}_{i}(x, f)=a_{i} \alpha_{i} f+\tilde{q}_{i}(x), \quad a_{i}=\frac{x_{i+1}-x_{i}}{x_{n}-x_{1}}, \\
\tilde{q}_{i}(x)=\tilde{f}_{i}-a_{i} \alpha_{i} \tilde{f}_{1}+a_{i} \int_{x_{1}}^{x} \frac{U_{i, M}(\tau)}{V_{i, N}(\tau)} d \tau \\
\left(\text { or } \tilde{q}_{i}(x)=\tilde{f}_{i+1}-a_{i} \alpha_{i} \tilde{f}_{1}-a_{i} \int_{x}^{x_{n}} \frac{U_{i, M}(\tau)}{V_{i, N}(\tau)} d \tau\right), \\
\tilde{f}_{i}=\tilde{f}_{1}+\sum_{r=1}^{i} a_{r}\left(\alpha_{r}\left(\tilde{f}_{n}-\tilde{f}_{1}\right)+\int_{x_{1}}^{x_{n}} \frac{U_{r, M}(\tau)}{V_{r, N}(\tau)} d \tau\right) \\
\text { or } \left.\tilde{f}_{i}=\tilde{f}_{n}-\sum_{r=i}^{n} a_{r}\left[\alpha_{r}\left(\tilde{f}_{n}-\tilde{f}_{1}\right)+\int_{x_{1}}^{x_{n}} \frac{U_{r, M}(\tau)}{V_{r, N}(\tau)} d \tau\right]\right), \\
\tilde{f}_{n}=\tilde{f}_{1}+\frac{i=2,3, \ldots, n-1,}{1-\sum_{r=1}^{n-1} a_{r} \int_{x_{1}}^{x_{n}} U_{r, M}(\tau) / V_{r, N}(\tau) d \tau} a_{r} \alpha_{r}
\end{gathered}
$$

Proposition 2. Let $\widetilde{\Phi}$ be the rational fractal function defined by (11) for a rational FIF $\Phi$ with $L_{i}(x)$ and $F_{i}(x, f)$ given by (10). Then $d \widetilde{\Phi}(x) / d x=\Phi(x)$ if and only if $\widetilde{\Phi}$ is the FIF related to IFS $\left\{I \times K^{*} ; \widetilde{w}_{i}(x, f)=\left(L_{i}(x), \widetilde{F}_{i}(x, f)\right), i=1,2, \ldots, n-1\right\}$, where $\widetilde{F}_{i}(x, f)$ is as defined above.

Since $V_{i, N}(x) \neq 0$ for all $x \in I$, the proof of the proposition follows through suitable modifications of the arguments in [6].

Examples 2. A rational FIF $\Phi$ is constructed in Figure 2(a) for $\{(0,0),(1 / 2,1),(3 / 4,-1),(1,0)\}$ with a choice of $\alpha_{i}=$ $0.75, i=1,2,3$. Suppose that $M=N=2, V_{i, 2}=1+x^{2}, U_{i, 2}=$ $c_{i}+d_{i} x+x^{2}$, where $c_{i}$ and $d_{i}$ are suitable real parameters. The values of $c_{i}$ and $d_{i}$ are calculated from (2), and we obtain

$$
\begin{aligned}
& L_{1}(x)=\frac{1}{2} x, \quad F_{1}(x, f)=\alpha_{1} f+\frac{x+x^{2}}{1+x^{2}} \\
& L_{2}(x)=\frac{1}{2}+\frac{1}{4} x, \quad F_{2}(x, f)=\alpha_{2} f+\frac{1+x+x^{2}}{1+x^{2}}, \\
& L_{3}(x)=\frac{3}{4}+\frac{1}{4} x, \quad F_{3}(x, f)=\alpha_{3} f+\frac{-1-4 x+x^{2}}{1+x^{2}} .
\end{aligned}
$$

The iteration of the IFS $\left\{C ;\left(L_{i}(x), F_{i}(x, f)\right), i=1,2,3\right\}$ generates the rational FIF $\Phi$ (see Figure 2(a)). Choosing $\tilde{f}_{1}=0, \widetilde{\Phi}(x)=\int_{0}^{x} \Phi(\tau) d \tau$ interpolates a new set of data $\{(0,0),(1 / 2,0.3426),(3 / 4,0.277),(1,0.1653)\}$ (see Figure $2(\mathrm{~b}))$. The IFS of $\widetilde{\Phi}$ contains the same $L_{i}(x)$ for $i=$ $1,2,3$, and

$$
\begin{aligned}
\widetilde{F}_{1}(x, f)= & \frac{3}{8} f-0.5 \tan ^{-1} x+0.25 \log \left(1+x^{2}\right) \\
& +\frac{x}{2}-0.4539, \\
\widetilde{F}_{2}(x, f)= & \frac{3}{16} f-\log \left(1+x^{2}\right)+\frac{x}{4}+1.4789, \\
\widetilde{F}_{3}(x, f)= & \frac{3}{16} f+0.5 \tan ^{-1} x+\frac{x}{4}+0.4197 .
\end{aligned}
$$

Similarly, for $\tilde{f}_{n}=0, \widetilde{\Phi}(x)=-\int_{x}^{1} \Phi(\tau) d \tau$ interpolates the data $\{(0,-0.1653),(1 / 2,0.1773),(3 / 4,0.1117),(1,0)\}$ (see Figure $2(\mathrm{c}))$. In this case, the IFS contains the same $L_{i}(x)$ for $i=1,2,3$, and

$$
\begin{aligned}
\widetilde{F}_{1}(x, f)= & \frac{3}{8} f-0.5 \tan ^{-1} x+0.25 \log \left(1+x^{2}\right) \\
& +\frac{x}{2}-0.6192, \\
\widetilde{F}_{2}(x, f)= & \frac{3}{16} f-\log \left(1+x^{2}\right)+\frac{x}{4}+1.3136, \\
\widetilde{F}_{3}(x, f)= & \frac{3}{16} f+0.5 \tan ^{-1} x+\frac{x}{4}+0.1427 .
\end{aligned}
$$

The functional values of $\widetilde{\Phi}$ in Figure $2(b)$ and $\widetilde{\Phi}$ in Figure 2(c) differ by 0.1653 at every $x \in[0,1]$, whereas the $\widetilde{F}_{i}(x, f), i=1,2,3$, in the IFSs of Figures 2(b) and 2(c) differ by three different constants.

Based on Proposition 2, it is clear that the scaling factors are restricted to certain ranges for a differentiable rational FIF. If $q_{i}^{(m)}(x)$ exists and is continuous for $i=1,2, \ldots, n-1$ and $m=0,1, \ldots, p$, that is, $V_{i, N}(x) \neq 0 \forall x \in I$, then we have the following theorem based on the join-up conditions for $F_{i}^{m}$.

Theorem 3. Let $\left\{\left(x_{i}, f_{i}\right): i=1,2, \ldots, n\right\}$ be a given data set. Suppose that $L_{i}(x)=a_{i} x+b_{i}, F_{i}(x, f)=\alpha_{i} f+$ $q_{i}(x), q_{i}(x)=U_{i, M}(x) / V_{i, N}(x), U_{i, M}(x), V_{i, N}(x)$ are suitably chosen polynomials in $x$ of degree $M, N$, respectively, and $V_{i, N}(x) \neq 0$ for every $x \in I$. Suppose for some integer $p \geq 0$, $\left|\alpha_{i}\right|<a_{i}^{p}, i=1,2, \ldots, n-1$. Let

$$
F_{i}^{m}(x, f)=\frac{\alpha_{i} f+q_{i}^{(m)}(x)}{a_{i}^{m}},
$$

where $q_{i}^{(m)}(x)$ represents the $m$ th derivative of $q_{i}(x)$,

$$
f_{1}^{m}=\frac{q_{1}^{(m)}\left(x_{1}\right)}{a_{1}^{m}-\alpha_{1}}, \quad f_{n}^{m}=\frac{q_{n-1}^{(m)}\left(x_{n}\right)}{a_{n-1}^{m}-\alpha_{n-1}}, \quad m=1,2, \ldots, p .
$$




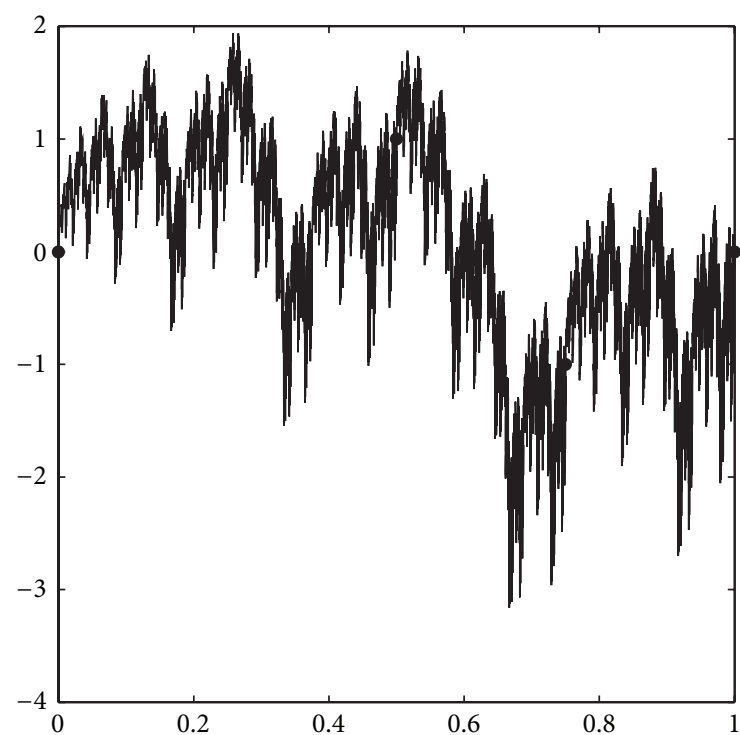

- Interpolation data $\phi$

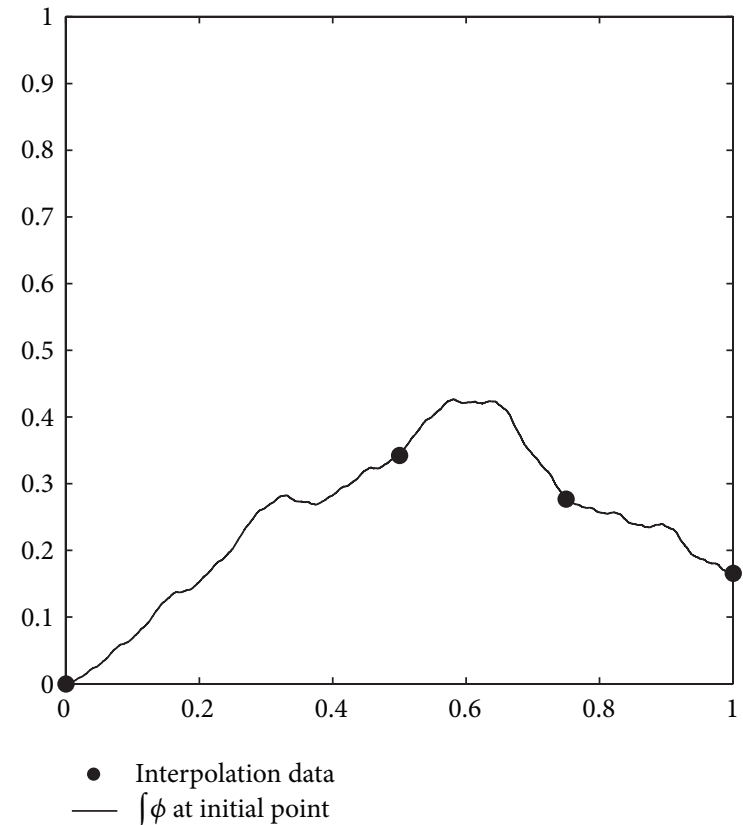

(b) $\widetilde{\Phi}$ with $\tilde{f}_{1}=0$

(a) Rational FIF $\Phi$

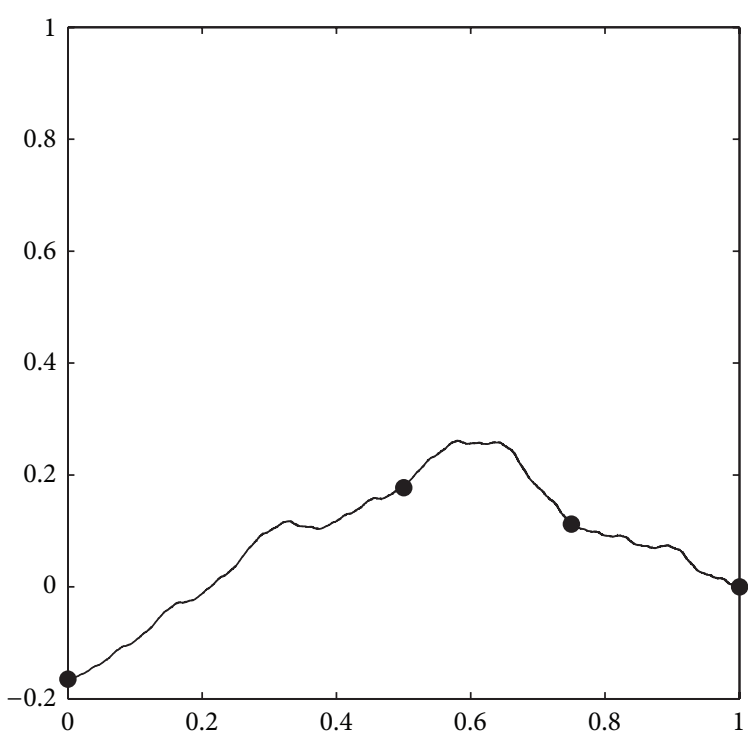

- Interpolation data $\int \phi$ at initial point

$$
\text { (c) } \widetilde{\Phi} \text { with } \tilde{f}_{n}=0
$$

FIGURE 2: Rational FIF and its integrals

If $F_{i}^{m}\left(x_{n}, f_{n}^{m}\right)=F_{i+1}^{m}\left(x_{1}, f_{1}^{m}\right), i=1,2, \ldots, n-2$, $m=1,2, \ldots, p$; then $\left\{C ; w_{i}(x, f)=\left(L_{i}(x), F_{i}(x, f)\right), i=\right.$ $1,2, \ldots, n-1\}$ determines a rational FIF $\Phi \quad \epsilon$ $\mathscr{C}^{P}(I)$, and $\Phi^{(m)}$ is the rational FIF determined by $\left\{I \times K_{m} ; w_{i}(x, f)=\left(L_{i}(x), F_{i}^{m}(x, f)\right), i=1,2, \ldots, n-1\right\}$ for $m=1,2, \ldots, p$, and $K_{m}$ is a suitable compact subset of $\mathbb{R}$.

The proof of the above theorem follows from the inductive arguments based on Proposition 2

\section{Rational Quadratic FIFs}

The principle of construction and evaluation of a $\mathscr{C}^{1}$-rational quadratic FIF are described in Sections 3.1 and 3.2, respectively.

In our construction of the rational quadratic FIFs, it is assumed that $q_{i}(i=1,2, \ldots, n-1)$ are the rational quadratic functions, where both the numerators and denominators are polynomials in $x$ of degree 2 ; that is, $M=N=2$. 


\subsection{Principle of Construction of $\mathscr{C}^{1}$-Rational Quadratic FIFs}

Theorem 4. Let $\left\{\left(x_{i}, f_{i}\right), i=1,2, \ldots, n\right\}$ be a given data set, where $x_{1}<x_{2}<\cdots<x_{n}$, and let $d_{i}(i=$ $1,2, \ldots, n)$ be the derivative values at the knots. Consider the IFS $\mathscr{I}^{*}=\left\{C ; w_{i}(x, f)=\left(L_{i}(x), F_{i}(x, f)\right), i=1,2, \ldots, n-\right.$ $1\}$, where $L_{i}(x)=a_{i} x+b_{i}$ satisfies $(1), F_{i}(x, f)=\alpha_{i} f+$ $q_{i}(x), q_{i}(x)=U_{i, 2}(x) / V_{i, 2}(x)$ contains four real parameters, $U_{i, 2}(x)$ and $V_{i, 2}(x)$ are polynomials of degree $2, V_{i, 2}(x) \neq 0 \forall x \in$ $I$, and $\left|\alpha_{i}\right|<a_{i}, i=1,2, \ldots, n-1$. Let $F_{i}^{1}(x, f)=\left(\alpha_{i} f+\right.$ $\left.q_{i}^{(1)}(x)\right) / a_{i}$, where $q_{i}^{(1)}(x)$ represents the derivative of $q_{i}(x)$ with respect to $x$. If, for $i=1,2, \ldots, n-1$,

$$
\begin{array}{ll}
F_{i}\left(x_{1}, f_{1}\right)=f_{i}, & F_{i}\left(x_{n}, f_{n}\right)=f_{i+1} \\
F_{i}^{1}\left(x_{1}, d_{1}\right)=d_{i}, & F_{i}^{1}\left(x_{n}, d_{n}\right)=d_{i+1},
\end{array}
$$

then the attractor of the IFS $\mathscr{I}^{*}$ is the graph of a $\mathscr{C}^{1}$-rational quadratic FIF.

Proof. Suppose that $\mathscr{F}^{*}=\{g: I \rightarrow \mathbb{R}$ | $g, g^{\prime}$ are continuous, $g\left(x_{1}\right)=f_{1}$, and $\left.g\left(x_{n}\right)=f_{n}\right\}$. Let $\rho^{*}$ be the metric on $\mathscr{F}^{*}$ induced by the $\mathscr{C}^{1}$-norm on $\mathscr{C}^{1}(I)$. Then $\left(\mathscr{F}^{*}, \rho^{*}\right)$ is a complete metric space. Define the ReadBajraktarević operator $T^{*}$ on $\mathscr{F}^{*}$ as

$$
\begin{array}{r}
T^{*} g(x)=\alpha_{i} g\left(L_{i}^{-1}(x)\right)+q_{i}\left(L_{i}^{-1}(x)\right), \\
x \in I_{i}, i=1,2 \ldots, n-1 .
\end{array}
$$

Since $a_{i}=\left(x_{i+1}-x_{i}\right) /\left(x_{n}-x_{1}\right)<1$, the conditions $\left|\alpha_{i}\right|<a_{i}<1$ and (19) give that $T^{*}$ is a contractive operator on $\left(\mathscr{F}^{*}, \rho^{*}\right)$. The fixed point $r \in \mathscr{C}^{1}(I)$ of $T^{*}$ is a fractal function that satisfies the functional relation

$$
r\left(L_{i}(x)\right)=\alpha_{i} r(x)+q_{i}(x), \quad x \in I, i=1,2 \ldots, n-1 .
$$

The four parameters in the rational quadratic function $q_{i}$ are evaluated by using the interpolation conditions (18) of $r$ as follows. Substituting $x=x_{1}$ and $x=x_{n}$ in (20), we get two equations involving $f_{i}$ and $f_{i+1}$, respectively, as

$$
f_{i}=\alpha_{i} f_{1}+q_{i}\left(x_{1}\right), \quad f_{i+1}=\alpha_{i} f_{n}+q_{i}\left(x_{n}\right) .
$$

Since $r \in \mathscr{C}^{1}(I), r^{(1)}$ satisfies the functional equation

$$
\begin{array}{r}
r^{(1)}\left(L_{i}(x)\right)=\frac{\alpha_{i} r^{(1)}(x)}{a_{i}}+\frac{q_{i}^{(1)}(x)}{a_{i}}, \\
x \in I, \quad i=1,2 \ldots, n-1 .
\end{array}
$$

Since $\left|\alpha_{i}\right| / a_{i}<1$, and $V_{i, 2}(x) \neq 0 \forall x \in I$ for $i=1,2, \ldots, n-1$, it is easy to verify that $r^{(1)}$ is a fractal function. Substituting $x=x_{1}$ and $x=x_{n}$ in (22), we have two equations involving $d_{i}$ and $d_{i+1}$, respectively, as

$$
d_{i}=\frac{\alpha_{i} d_{1}}{a_{i}}+\frac{q_{i}^{(1)}\left(x_{1}\right)}{a_{i}}, \quad d_{i+1}=\frac{\alpha_{i} d_{n}}{a_{i}}+\frac{q_{i}^{(1)}\left(x_{n}\right)}{a_{i}} .
$$

When the four parameters of $q_{i}$ are determined from (21) and (23), then the rational quadratic FIF exists. By using similar arguments as in [1], it can be shown that the IFS $\mathscr{I}^{*}$ has a unique attractor, and it is the graph of the rational quadratic FIF $r \in \mathscr{C}^{1}(I)$.

Remark 5. The function $r \in \mathscr{C}^{1}(I)$ is called a fractal function because of (i) the presence of the scaling vector $\alpha$ in (20), (ii) the derivative $r^{\prime}$ is a typically fractal function, and (iii) the graph of $r$, say $G_{r}$, satisfies the equation: $G_{r}=\cup_{i=1}^{n-1} w_{i}\left(G_{r}\right)$.

3.2. Evaluation of $\mathscr{C}^{1}$-Rational Quadratic FIFs. Denote $\Delta_{i}=$ $\left(f_{i+1}-f_{i}\right) /\left(x_{i+1}-x_{i}\right), \theta=\left(x-x_{1}\right) /\left(x_{n}-x_{1}\right), x \in I$. Assume that

$$
\begin{array}{r}
U_{i, 2}(\theta):=X_{i} \theta^{2}+Y_{i} \theta(1-\theta)+Z_{i}(1-\theta)^{2}, \\
V_{i, 2}(\theta):=\Delta_{i} \theta^{2}+W_{i} \theta(1-\theta)+\Delta_{i}(1-\theta)^{2} \neq 0, \\
i=1,2, \ldots, n-1,
\end{array}
$$

where $X_{i}, Y_{i}, Z_{i}, W_{i}(i=1,2, \ldots, n-1)$ are suitable real parameters. By considering Theorem 4 with the above $U_{i, 2}(\theta)$ and $V_{i, 2}(\theta)$, we get the following functional equation:

$$
\begin{aligned}
& r\left(L_{i}(x)\right) \\
& = \begin{cases}\alpha_{i} r(x)+\frac{X_{i} \theta^{2}+Y_{i} \theta(1-\theta)+Z_{i}(1-\theta)^{2}}{\Delta_{i} \theta^{2}+W_{i} \theta(1-\theta)+\Delta_{i}(1-\theta)^{2}} & \text { if } \Delta_{i} \neq 0, \\
f_{i} & \text { if } \Delta_{i}=0 .\end{cases}
\end{aligned}
$$

The parameters $X_{i}, Y_{i}, Z_{i}, W_{i}(i=1,2, \ldots, n-1)$ are evaluated using the following interpolatory properties; namely,

$$
\begin{gathered}
r\left(x_{i}\right)=f_{i}, \quad r\left(x_{i+1}\right)=f_{i+1}, \quad r^{(1)}\left(x_{i}\right)=d_{i}, \\
r^{(1)}\left(x_{i+1}\right)=d_{i+1} .
\end{gathered}
$$

If $\Delta_{i}=0$, the rational quadratic FIF $r$ reduces to a constant in the subinterval $I_{i}$ with $f_{i}$ as in (25). Otherwise we proceed as follows.

By taking $x=x_{1},(25)$ gives that

$$
r\left(L_{i}\left(x_{1}\right)\right)=\alpha_{i} r\left(x_{1}\right)+\frac{Z_{i}}{\Delta_{i}} \Longrightarrow Z_{i}=\Delta_{i}\left(f_{i}-\alpha_{i} f_{1}\right) .
$$

Similarly, $x=x_{n}$ in (25) gives that

$$
r\left(L_{i}\left(x_{n}\right)\right)=\alpha_{i} r\left(x_{n}\right)+\frac{X_{i}}{\Delta_{i}} \Longrightarrow X_{i}=\Delta_{i}\left(f_{i+1}-\alpha_{i} f_{n}\right) .
$$

Using $r^{(1)}\left(x_{i}\right)=d_{i}, r^{(1)}\left(x_{i+1}\right)=d_{i+1}$ in (25), we have the coupled equations

$$
\begin{gathered}
\Delta_{i} Y_{i}-Z_{i} W_{i}-\Delta_{i}^{2}\left(x_{n}-x_{1}\right)\left(a_{i} d_{i}-\alpha_{i} d_{1}\right)=0, \\
X_{i} W_{i}-\Delta_{i} Y_{i}-\Delta_{i}^{2}\left(x_{n}-x_{1}\right)\left(a_{i} d_{i+1}-\alpha_{i} d_{n}\right)=0 .
\end{gathered}
$$




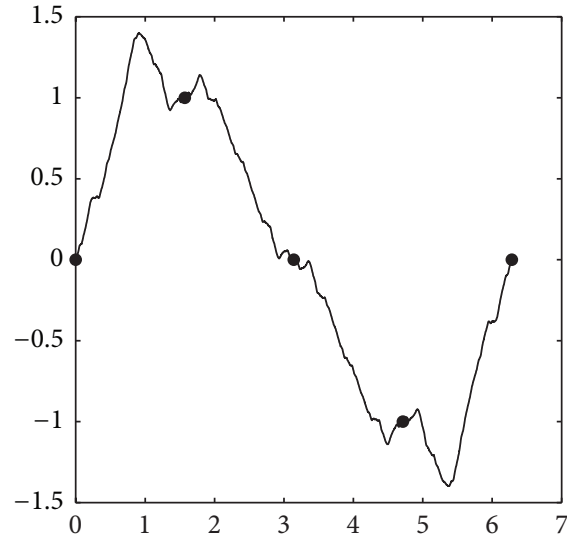

(a) $\alpha_{i}=0.2, i=1,2,3,4$

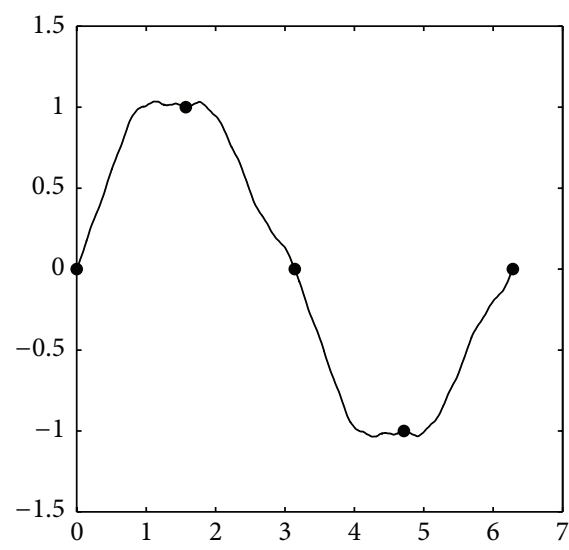

(c) $\alpha_{1}=\alpha_{2}=-\alpha_{3}=-\alpha_{4}=0.14$

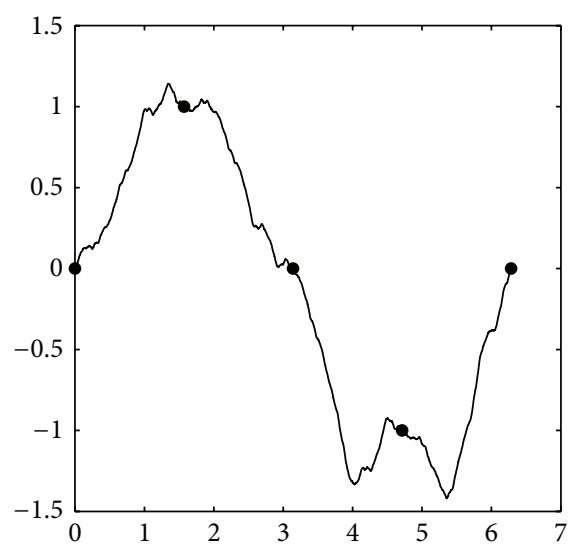

- Interpolation data

Rational FIF

(e) $\alpha_{1}=\alpha_{3}=-0.2, \alpha_{2}=\alpha_{4}=0.2$

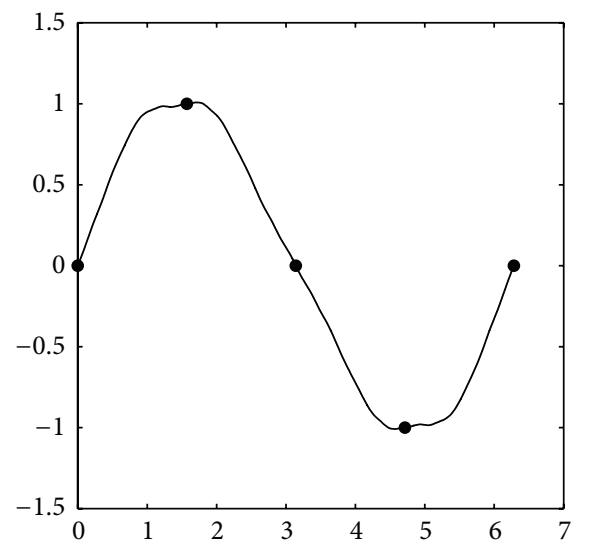

(b) $\alpha_{i}=0.1, i=1,2,3,4$

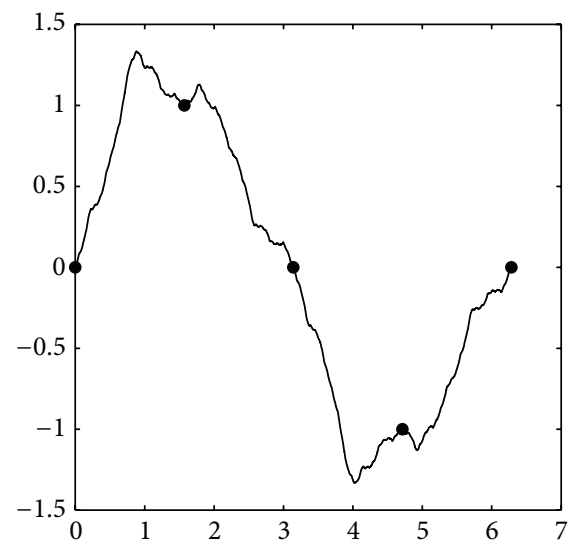

(d) $\alpha_{1}=\alpha_{2}=0.2, \alpha_{3}=\alpha_{4}=-0.2$

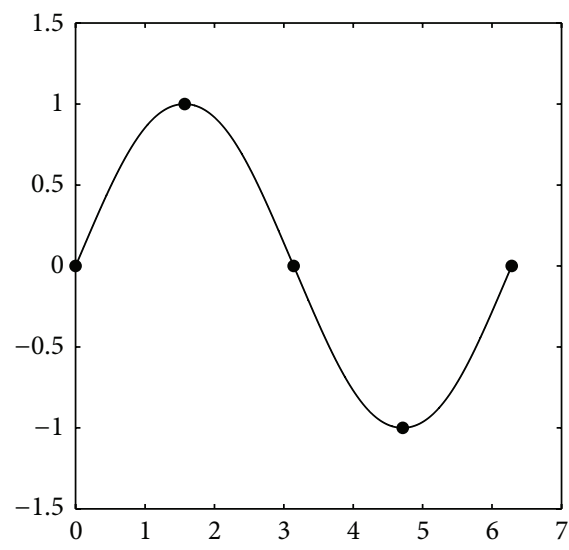

- Interpolation data

- Rational FIF

(f) $\alpha_{1}=\alpha_{2}=\alpha_{3}=\alpha_{4}=0$

FIGURE 3: Rational quadratic FIFs for sine function. 
Assume that $f_{i+1}-f_{i}-\alpha_{i}\left(f_{n}-f_{1}\right) \neq 0$. The solution of the system (29) gives that

$$
\begin{aligned}
W_{i}=\beta_{i}[ & \left.a_{i}\left(d_{i}+d_{i+1}\right)-\alpha_{i}\left(d_{1}+d_{n}\right)\right], \\
Y_{i}=\beta_{i}\left[\left(f_{i} d_{i+1}+f_{i+1} d_{i}\right) a_{i}\right. & \\
& -\alpha_{i}\left(f_{i+1} d_{1}+f_{i} d_{n}+f_{n} d_{i} a_{i}+f_{1} d_{i+1} a_{i}\right) \\
& \left.+\alpha_{i}^{2}\left(f_{n} d_{1}+f_{1} d_{n}\right)\right],
\end{aligned}
$$

where $\beta_{i}=\Delta_{i}\left(x_{n}-x_{1}\right) /\left(f_{i+1}-f_{i}-\alpha_{i}\left(f_{n}-f_{1}\right)\right)$. Substituting the values of $W_{i}, X_{i}, Y_{i}, Z_{i}$ in (25), the desired rational quadratic FIF is finally obtained as

$$
\begin{aligned}
& r\left(L_{i}(x)\right)= \begin{cases}\alpha_{i} r(x)+\frac{U_{i, 2}(\theta)}{V_{i, 2}(\theta)} & \text { if } \Delta_{i} \neq 0, \\
f_{i} & \text { if } \Delta_{i}=0\end{cases} \\
& U_{i, 2}(\theta) \\
& =\left(f_{i+1}-\alpha_{i} f_{n}\right) \Delta_{i} \theta^{2} \\
& +\beta_{i}\left[\left(f_{i} d_{i+1}+f_{i+1} d_{i}\right) a_{i}\right. \\
& -\alpha_{i}\left\{f_{i+1} d_{1}+f_{i} d_{n}+a_{i}\left(f_{n} d_{i}+f_{1} d_{i+1}\right)\right\} \\
& \left.+\alpha_{i}^{2}\left(f_{n} d_{1}+f_{1} d_{n}\right)\right] \theta(1-\theta) \\
& +\left(f_{i}-\alpha_{i} f_{1}\right)(1-\theta)^{2} \Delta_{i} \\
& V_{i, 2}(\theta)=\Delta_{i} \theta^{2}+\beta_{i}\left[a_{i}\left(d_{i}+d_{i+1}\right)-\alpha_{i}\left(d_{1}+d_{n}\right)\right] \theta \\
& \times(1-\theta)+\Delta_{i}(1-\theta)^{2} .
\end{aligned}
$$

If $\Delta_{i}=0$, then $V_{i, 2}(\theta)=0$. In order to avoid singularity in the expression of the rational quadratic FIF $r$, we define $r$ as a constant function with the value $f_{i}$ in the subinterval $I_{i}$. In general, $V_{i, 2}(\theta)$ need not be nonzero for all $\theta \in[0,1]$. But the objective of this paper is to obtain monotonic curves though the rational quadratic FIF $r$ for given monotonic data, and when we impose the sufficient conditions for monotonicity based on the derivative values and scaling factors, automatically we get $V_{i, 2}(\theta) \neq 0$ for all $\theta \in[0,1]$. This is explained in Section 4.

In most applications, the derivatives $d_{i}(i=1,2, \ldots, n)$ are not given and hence must be calculated either from the given data or some numerical methods. In this paper, they are computed from the given data in such a way that the $\mathscr{C}^{1}$-smoothness of the fractal interpolant (31) is retained. These methods are different type of approximations based on the various mathematical theories in the literature; see, for instance, [42].

We use the following approximations for the shape preserving rational quadratic FIFs.
3.2.1. Arithmetic Mean Method. The three-point difference method is used to approximate the derivatives at the intermediate nodes as

$$
d_{i}= \begin{cases}0 & \text { if } \Delta_{i-1}=0 \text { or } \Delta_{i}=0, \\ \frac{h_{i} \Delta_{i-1}+h_{i-1} \Delta_{i}}{h_{i}+h_{i-1}} & \text { otherwise, } i=2,3, \ldots, n-1,\end{cases}
$$

and at the end points, we have

$$
\begin{aligned}
& d_{1}=\left\{\begin{array}{l}
0 \\
\text { if } \Delta_{1}=0 \text { or } \operatorname{sgn}\left(d_{1}^{*}\right) \neq \operatorname{sgn}\left(\Delta_{1}\right), \\
d_{1}^{*}=\Delta_{1}+\frac{\left(\Delta_{1}-\Delta_{2}\right) h_{1}}{h_{1}+h_{2}} \\
\text { otherwise, }
\end{array}\right. \\
& d_{n}=\left\{\begin{array}{c}
0 \\
\text { if } \Delta_{n-1}=0 \text { or } \operatorname{sgn}\left(d_{n}^{*}\right) \neq \operatorname{sgn}\left(\Delta_{n-1}\right), \\
d_{1}^{*}=\Delta_{n-1}+\frac{\left(\Delta_{n-1}-\Delta_{n-2}\right) h_{n-1}}{h_{n-1}+h_{n-2}} \\
\text { otherwise. }
\end{array}\right.
\end{aligned}
$$

3.2.2. Geometric Mean Method. The nonlinear approximation method is used to approximate the derivatives at the intermediate nodes as

$$
d_{i}= \begin{cases}0 & \text { if } \Delta_{i-1}=0 \text { or } \Delta_{i}=0, \\ \Delta_{i-1}^{h_{i} /\left(h_{i}+h_{i-1}\right)} \Delta_{i}^{h_{i-1} /\left(h_{i}+h_{i-1}\right)} & \text { otherwise, } i=2,3, \ldots, n-1,\end{cases}
$$

and at the end points, we have

$$
\begin{aligned}
& d_{1}= \begin{cases}0 & \text { if } \Delta_{1}=0 \text { or } \Delta_{3,1}=0, \\
\Delta_{1}\left(\frac{\Delta_{1}}{\Delta_{3,1}}\right)^{h_{1} / h_{2}} & \text { otherwise, }\end{cases} \\
& d_{n}= \begin{cases}0 & \text { if } \Delta_{n-1}=0 \text { or } \Delta_{n, n-2}=0, \\
\Delta_{n-1}\left(\frac{\Delta_{n-1}}{\Delta_{n, n-2}}\right)^{h_{n-1} / h_{n-2}} & \text { otherwise, }\end{cases}
\end{aligned}
$$

where $\Delta_{3,1}=\left(f_{3}-f_{1}\right) /\left(x_{3}-x_{1}\right), \Delta_{n, n-2}=\left(f_{n}-f_{n-2}\right) /\left(x_{n}-\right.$ $\left.x_{n-2}\right)$.

For a given bounded data set, the above derivative approximations are bounded.

Remark 6. Let $\left\{\left(x_{i}, f_{i}\right), i=1,2, \ldots, n\right\}$ be a given data set, where $x_{1}<x_{2}<\cdots<x_{n}$, and let $d_{i}(i=1,2, \ldots, n)$ be the derivative values given or approximated (by the abovementioned methods) at the knots. If $\left|\alpha_{i}\right|<a_{i}, V_{i, 2}(\theta) \neq 0$ for all $\theta \in[0,1], i=1,2, \ldots, n-1$, then the rational quadratic FIF $r$ (cf. (25)) is in $\mathscr{C}^{1}(I)$ and is unique for fixed $\alpha_{i}, i=1,2, \ldots, n-$ 1 , such that $r\left(x_{i}\right)=f_{i}$ and $r^{(1)}\left(x_{i}\right)=d_{i}, i=1,2 \ldots, n$. 
TABLE 1: Parameters of the rational quadratic IFSs for sine function.

\begin{tabular}{lccccc}
\hline Figure 3(a) & Figure 3(b) & Figure 3(c) & Figure 3(d) & Figure 3(e) & Figure 3(f) \\
\hline$W_{1}=-0.6$ & $W_{1}=0.2$ & $W_{1}=-0.12$ & $W_{1}=-0.6$ & $W_{1}=2.6$ & $W_{1}=1$ \\
$X_{1}=1$ & $X_{1}=1$ & $X_{1}=0$ & $X_{1}=0$ & $X_{1}=0$ & $X_{1}=0$ \\
$Y_{1}=0.2$ & $Y_{1}=0.6$ & $Y_{1}=0.44$ & $Y_{1}=0.2$ & $Y_{1}=1.8$ & $Y_{1}=1$ \\
$Z_{1}=0$ & $Z_{1}=0$ & $Z_{1}=0$ & $Z_{1}=0$ & $Z_{1}=0$ & $Z_{1}=0$ \\
\hline$W_{2}=2.6$ & $W_{2}=1.8$ & $W_{2}=2.12$ & $W_{2}=2.6$ & $W_{2}=2.6$ & $W_{2}=1$ \\
$X_{2}=0$ & $X_{2}=0$ & $X_{2}=1$ & $X_{2}=1$ & $X_{2}=1$ & $X_{2}=1$ \\
$Y_{2}=1.8$ & $Y_{2}=1.4$ & $Y_{2}=1.56$ & $Y_{2}=1.8$ & $Y_{2}=1.8$ & $Y_{2}=1$ \\
$Z_{2}=1$ & $Z_{2}=1$ & $Z_{2}=1$ & $Z_{2}=1$ & $Z_{2}=1$ & $Z_{2}=1$ \\
\hline$W_{3}=2.6$ & $W_{3}=1.8$ & $W_{3}=-0.12$ & $W_{3}=-0.6$ & $W_{3}=-0.6$ & $W_{3}=1$ \\
$X_{3}=-1$ & $X_{3}=-1$ & $X_{3}=-1$ & $X_{3}=-1$ & $X_{3}=-1$ & $X_{3}=-1$ \\
$Y_{3}=1.8$ & $Y_{3}=1.4$ & $Y_{3}=0.44$ & $Y_{3}=0.2$ & $Y_{3}=0.2$ & $Y_{3}=1$ \\
$Z_{3}=0$ & $Z_{3}=0$ & $Z_{3}=0$ & $Z_{3}=0$ & $Z_{3}=0$ & $Z_{3}=0$ \\
\hline$W_{4}=-0.6$ & $W_{4}=0.2$ & $W_{4}=2.12$ & $W_{4}=2.6$ & $W_{4}=-0.6$ & $W_{4}=1$ \\
$X_{4}=0$ & $X_{4}=0$ & $X_{4}=0$ & $X_{4}=0$ & $X_{4}=0$ & $X_{4}=0$ \\
$Y_{4}=0.2$ & $Y_{4}=0.6$ & $Y_{4}=1.56$ & $Y_{4}=1.8$ & $Y_{4}=0.2$ & $Y_{4}=1$ \\
$Z_{4}=-1$ & $Z_{4}=-1$ & $Z_{4}=-1$ & $Z_{4}=-1$ & $Z_{4}=-1$ & $Z_{4}=-1$ \\
\hline
\end{tabular}

Remark 7. If $\alpha_{i}=0, i=1,2, \ldots, n-1$, the rational quadratic FIF (31) is reduced to the classical rational quadratic interpolation function $S$ [32]. Using the notation $\rho=(x-$ $\left.x_{i}\right) /\left(x_{i+1}-x_{i}\right), S$ is given by

$$
S(x)= \begin{cases} & \left(\Delta_{i} f_{i+1} \rho^{2}+\left(f_{i} d_{i+1}+f_{i+1} d_{i}\right) \rho(1-\rho)\right. \\ & \left.+\Delta_{i} f_{i}(1-\rho)^{2}\right) \\ \times\left(\Delta_{i} \rho^{2}+\left(d_{i}+d_{i+1}\right) \rho\right. & \left.(1-\rho)+\Delta_{i}(1-\rho)^{2}\right)^{-1} \\ & \text { if } \Delta_{i} \neq 0 \\ f_{i} & \text { if } \Delta_{i}=0\end{cases}
$$

Examples 3. We construct six different $\mathscr{C}^{1}$-rational quadratic fractal interpolants for the data set $\{(0,0),(\pi / 2,1),(\pi, 0)$, $(3 \pi / 2,-1),(2 \pi, 0)\}$ of sine function with six different sets of scaling factors, where it is given that $d_{1}=1, d_{2}=0$, $d_{3}=-1, d_{4}=0$, and $d_{5}=1$. Using (6) in our examples, $L_{1}(x)=(1 / 4) x, L_{2}(x)=(1 / 4) x+(\pi / 2), L_{3}(x)=(1 / 4) x+\pi$, and $L_{4}(x)=(1 / 4) x+(3 \pi / 2)$. Depending on the scaling factors (see Table 1), the corresponding parameters $X_{i}, Y_{i}, Z_{i}$, $W_{i}, i=1,2,3,4$, are determined according to the procedure described in Section 3.2. Using the IFS

$$
\begin{gathered}
\left\{C ; w_{i}(x, f)=\left(L_{i}(x), F_{i}(x, f)\right), i=1,2,3,4\right\} \\
\text { with } \theta=\frac{L_{i}^{-1}(x)-x_{1}}{x_{n}-x_{1}}, \\
F_{i}(x, f)=\left\{\begin{array}{rr}
\alpha_{i} f+\left(X_{i} \Delta_{i} \theta^{2}+Y_{i}\left(f_{i} d_{i+1}+f_{i+1} d_{i}\right)\right. \\
\left.\times \theta(1-\theta)+Z_{i} \Delta_{i}(1-\theta)^{2}\right) \\
\times\left(\Delta_{i} \theta^{2}+W_{i}\left(d_{i+1}+d_{i}\right) \theta(1-\theta)\right. \\
\left.+\Delta_{i}(1-\theta)^{2}\right)^{-1} & \text { if } \Delta_{i} \neq 0, \\
f_{i} & \text { if } \Delta_{i}=0,
\end{array}\right.
\end{gathered}
$$

the rational quadratic FIFs are generated iteratively (see Figures 3(a) and 3(f)). If we assume $\alpha_{i}=$ constant, then it is observed that $r(x)=-r(2 \pi-x), x \in[0, \pi]$, and the rational quadratic FIFs of sine curve have 2-fold symmetry about $x=\pi$ and $y=0$ (see Figures 3(a) and 3(b)). When $\alpha_{1}=\alpha_{2}=c \neq 0, \alpha_{3}=\alpha_{4}=-c$, then $r(x)=-r(\pi+x)$, and the rational quadratic FIFs are shifted 1-line of horizontal symmetry about $y=0$ (see Figures $3(\mathrm{c})$ and $3(\mathrm{~d})$ ). If $\alpha_{i}$ are alternatively the same, that is, $\alpha_{1}=\alpha_{3}=c \neq 0, \alpha_{2}=\alpha_{4}=-c$, then there is no relation between any part of the curve of a rational quadratic FIF (see Figure 3(e)), and this case is similar to the behavior of the rational quadratic FIFs when all $\alpha_{i}$ 's are unequal. When all scaling factors $\alpha_{i}=0(i=$ $1,2,3,4)$, the rational quadratic FIF reduces to the classical quadratic spline that is generated by using the IFS (37) given in Figure 3(f). These examples illustrate the importance of the scaling factors in the modeling of periodic curves, where different types of symmetries can be explored depending on various scientific and engineering applications.

\section{Monotonicity Preserving Fractal Interpolation}

The sufficient conditions are derived to preserve the monotonicity of a given monotonic data set in Section 4.1. The convergence results of a monotonic rational quadratic FIF to the original function $R \in \mathscr{C}^{4}(I)$ are deduced in Section 4.2.

\subsection{Sufficient Condition for Monotonic Rational Quadratic} FIF. The $\mathscr{C}^{1}$-rational quadratic FIF $r$ may not preserve the monotonicity property of given monotonic data if we simply assume all interpolatory conditions. To achieve the shape preserving property, namely, monotonicity, by the rational quadratic FIF $r$ for given monotonic data, we need some 
mathematical treatment that is based on the recursive nature of a FIF. The following theorem addresses this issue.

Theorem 8. Let $\left\{\left(x_{i}, f_{i}, d_{i}\right), i=1,2, \ldots, n\right\}$ be a given monotonic data set. Let the derivative values satisfy the necessary conditions for the monotonicity; namely,

$$
\begin{aligned}
& d_{i}=d_{i+1}=0 \text { for } \Delta_{i}=0, \\
& \operatorname{sgn}\left(d_{i}\right)=\operatorname{sgn}\left(d_{i+1}\right)=\operatorname{sgn}\left(\Delta_{i}\right) \text { for } \Delta_{i} \neq 0 .
\end{aligned}
$$

If the scaling factors $\alpha_{i}, i=1,2, \ldots, n-1$, are chosen in the following way:

$$
\alpha_{i} \in\left[0, \lambda_{i}\right), \quad i=1,2, \ldots, n-1,
$$

where $\lambda_{i}=\min \left\{a_{i} d_{i+1} / d_{n}, a_{i} d_{i} / d_{1},\left(f_{i+1}-f_{i}\right) /\left(f_{n}-f_{1}\right), a_{i}\right\}$, then the $\mathscr{C}^{1}$-rational quadratic FIF $r$ in (31) is monotonic over I.

Proof. From elementary calculus, the rational quadratic FIF $r$ is monotonic on $I$ if and only if either $r^{(1)}(x) \geq 0$ or $r^{(1)}(x) \leq$ 0 for every $x \in I$. Therefore, differentiating (31) with respect to $x$, and after some rigorous calculations, we get

$$
a_{i} r^{(1)}\left(L_{i}(x)\right)=\alpha_{i} r^{(1)}(x)+\frac{\Omega_{i}(x)}{\left(V_{i, 2}(\theta)\right)^{2}\left(x_{n}-x_{1}\right)},
$$

where

$$
\begin{aligned}
& \Omega_{i}(x)= T_{i, 1} \theta^{4}+\left(2 T_{i, 1}+T_{i, 2}\right) \theta^{3}(1-\theta) \\
&+\left(T_{i, 1}+T_{i, 3}\right) \theta^{2}(1-\theta)^{2} \\
&+\left(2 T_{i, 3}+T_{i, 2}\right) \theta(1-\theta)^{3}+T_{i, 3}(1-\theta)^{4}, \\
& T_{i, 1}=\Delta_{i} \beta_{i}\left[a_{i} d_{i+1}\left\{f_{i+1}-f_{i}-\alpha_{i}\left(f_{n}-f_{1}\right)\right\}\right. \\
&\left.-\alpha_{i} d_{n}\left\{f_{i+1}-f_{i}-\alpha_{i}\left(f_{n}-f_{1}\right)\right\}\right], \\
& T_{i, 2}=2 \Delta_{i}^{2}\left[f_{i+1}-f_{i}-\alpha_{i}\left(f_{n}-f_{1}\right)\right], \\
& T_{i, 3}=\Delta_{i} \beta_{i}\left[a_{i} d_{i}\left\{f_{i+1}-f_{i}-\alpha_{i}\left(f_{n}-f_{1}\right)\right\}\right. \\
&\left.-\alpha_{i} d_{1}\left\{f_{i+1}-f_{i}-\alpha_{i}\left(f_{n}-f_{1}\right)\right\}\right] .
\end{aligned}
$$

Due to the recursive nature of $r^{(1)}$ and the coefficients involved in $\Omega_{i}$ (cf. (40)), the necessary conditions (38) may not be sufficient to ensure the monotonicity of the rational quadratic FIF $r$. Therefore, we put restrictions on the scaling factors $\alpha_{i}$ so that these conditions together with the above necessary conditions give the monotonicity nature to our $\mathscr{C}^{1}$ rational quadratic FIF $r$ as follows.

Case I (monotonically decreasing data). In this case $f_{i} \geq f_{i+1}$, and hence $\Delta_{i}=\left(f_{i+1}-f_{i}\right) /\left(x_{i+1}-x_{i}\right) \leq 0$ for $i=1,2, \ldots, n-1$. It follows from (40) that $r$ is a monotonically decreasing rational quadratic FIF for a monotonically decreasing data if $\alpha_{i} \geq 0$ and $\Omega_{i}(x) \leq 0$ for $i=1,2, \ldots, n-1, x \in I$. For $\Omega_{i} \leq 0$ on $I$, the sufficient conditions are $T_{i, 1} \leq 0, T_{i, 2} \leq 0, T_{i, 3} \leq 0$ for $i=1,2, \ldots, n-1$. Since $r^{(1)}\left(x_{j}\right) \leq 0$ for $j=1,2, \ldots, n$, the first generation of points in the rational quadratic FIF $r^{(1)}\left(L_{i}\left(x_{j}\right)\right) \leq 0$ whenever $\alpha_{i} \geq 0$ and $T_{i, 1} \leq 0, T_{i, 2} \leq 0, T_{i, 3} \leq 0$ for $i=1,2, \ldots, n-1$. Consequently, $r^{(1)}(x) \leq 0$ for all $x \in I$ as $I$ is the attractor of the IFS $\left\{I ; L_{i}(x), i=1,2, \ldots, n-1\right\}$.

Suppose that $\Delta_{i}=0$ for any $i \in\{1,2, \ldots, n-1\}$; then $T_{i, 1}=T_{i, 2}=T_{i, 3}=0$. Thus, from $(40)$, we have $a_{i} r^{(1)}\left(L_{i}(x)\right)=$ $\alpha_{i} r^{(1)}(x)$ which is true if $r^{(1)}\left(L_{i}(x)\right)=0$ and $\alpha_{i}=0$ in (40); that is, in this case, the $\mathscr{C}^{1}$-rational quadratic FIF is a constant throughout the subinterval $I_{i}$ with the value $f_{i}$ and $\alpha_{i}=0$.

Again suppose that $\Delta_{i}<0$; that is, $\Delta_{i}^{2}>0$. With the assumption on $\alpha_{i}$ (see Section 3.2), substituting the value of $\beta_{i}$ in $T_{i, 1}$, and $T_{i, 3}$, we have $T_{i, 1}=\Delta_{i}^{2}\left[a_{i} d_{i+1}-\alpha_{i} d_{n}\right], T_{i, 3}=$ $\Delta_{i}^{2}\left[a_{i} d_{i}-\alpha_{i} d_{1}\right]$. Now

$$
\begin{gathered}
T_{i, 2}<0 \quad \text { if } f_{i+1}-f_{i}<\alpha_{i}\left(f_{n}-f_{1}\right) \Longleftrightarrow \alpha_{i}<\frac{f_{i+1}-f_{i}}{f_{n}-f_{1}}, \\
T_{i, 1} \leq 0 \quad \text { if }\left[a_{i} d_{i+1}-\alpha_{i} d_{n}\right] \leq 0 \Longleftrightarrow \alpha_{i} \leq \frac{a_{i} d_{i+1}}{d_{n}}, \\
T_{i, 3} \leq 0 \quad \text { if }\left[a_{i} d_{i}-\alpha_{i} d_{1}\right] \leq 0 \Longleftrightarrow \alpha_{i} \leq \frac{a_{i} d_{i}}{d_{1}} .
\end{gathered}
$$

From (42), $T_{i, 1} \leq 0, T_{i, 2}<0, T_{i, 3} \leq 0$ if

$$
\alpha_{i} \in\left[0, \mu_{i}\right), \quad i=1,2, \ldots, n-1,
$$

where $\mu_{i}=\min \left\{\left(a_{i} d_{i+1} / d_{n}\right),\left(a_{i} d_{i} / d_{1}\right),\left(f_{i+1}-f_{i}\right) /\left(f_{n}-f_{1}\right)\right\}$. Thus in this case, the $\mathscr{C}^{1}$-rational quadratic FIF is monotonically decreasing if the scaling factors $\alpha_{i}, i=1,2, \ldots, n-1$, are chosen according to (39).

Case II (monotonically increasing data). In this case $f_{i} \leq f_{i+1}$, and hence $\Delta_{i} \geq 0$ for $i=1,2, \ldots, n-1$. It is assumed that $\alpha_{i} \geq 0$ for $i=1,2, \ldots, n-1$. It follows from (40) that $r$ is a monotonically increasing rational quadratic FIF for a monotonically increasing data if $\Omega_{i}(x) \geq 0$ for all $x \in I$, that is sufficient to the conditions $T_{i, 1} \geq 0, T_{i, 2} \geq 0, T_{i, 3} \geq 0, \alpha_{i} \geq 0$ which imply that $r^{(1)}(x) \geq 0$ for all $x \in I$.

Suppose that $\Delta_{i}=0$; then according to discussions in Case I, the $\mathscr{C}^{1}$-rational quadratic FIF is a constant throughout the sub-interval $I_{i}$ with the value $f_{i}$ and $\alpha_{i}=0$.

Again suppose that $\Delta_{i}>0$, using similar arguments as in Case I;

$$
\begin{gathered}
T_{i, 2}>0 \quad \text { if } \alpha_{i}<\frac{f_{i+1}-f_{i}}{f_{n}-f_{1}}, \\
T_{i, 1} \geq 0 \quad \text { if } \alpha_{i} \leq \frac{a_{i} d_{i+1}}{d_{n}}, \\
T_{i, 3} \geq 0 \quad \text { if } \alpha_{i} \leq \frac{a_{i} d_{i}}{d_{1}} .
\end{gathered}
$$

From (44), $T_{i, 1} \geq 0, T_{i, 2}>0, T_{i, 3} \geq 0$ whenever (43) holds.

In this case, now it is easy to see that if the scaling factors $\alpha_{i}, i=1,2, \ldots, n-1$, are chosen according to (39), then the 
$\mathscr{C}^{1}$-rational quadratic FIF $r$ is monotonically increasing over I. Therefore, using the results from Case I and Case II, we conclude that the selection of the scaling factors according to (39) is sufficient to obtain the monotonic rational quadratic FIF $r$ for a given set of monotonic data. This completes the proof of Theorem 8 .

Remark 9. For a given set of monotonic data, if $\Delta_{i} \neq 0$ and the scaling factor $\alpha_{i}$ is chosen with respect to (39), then it is easy to observe that $\operatorname{sgn}\left(V_{i, 2}(\theta)\right)=\operatorname{sgn}\left(\Delta_{i}\right)$. Consequently, $V_{i, 2}(\theta) \neq 0$ for all $\theta \in[0,1]$.

\subsection{Uniform Error Bound for $\mathscr{C}^{1}$-Monotonic Rational Quadratic FIFs}

Theorem 10. Let $r, S$, respectively, be the monotonic rational quadratic FIF and the classical monotonic rational quadratic function with respect to a data set $\left\{\left(x_{i}, f_{i}\right), i=1,2, \ldots, n\right\}$ from the original function $R \in \mathscr{C}^{4}(I)$. Let the derivatives $d_{i}, i=1,2, \ldots, n$, satisfy the necessary conditions (38) for monotonicity. Suppose that $\mathscr{V}^{*}=\left[0, \kappa \lambda_{1}\right] \times\left[0, \kappa \lambda_{2}\right] \times \cdots \times$ $\left[0, \kappa \lambda_{n-1}\right]$, where $0 \leq \kappa<1$. If $\left|R^{(1)}(x)\right|>0$ on $I$; then

$$
\begin{aligned}
\|R-r\|_{\infty} \leq & h A(R) \max _{1 \leq i \leq n-1}\left\{\left|R_{i}^{(1)}-d_{i}\right|,\left|R_{i+1}^{(1)}-d_{i+1}\right|\right\} \\
& +h^{4} \max _{1 \leq i \leq n-1} B_{i}(R)+\frac{\alpha_{\infty}}{1-\alpha_{\infty}}(\Lambda+U(\alpha)),
\end{aligned}
$$

where $h=\max _{1 \leq i \leq n-1} h_{i}, f_{i}=R\left(x_{i}\right), R_{i}^{(k)}=R^{(k)}\left(x_{i}\right), k=$ $1,2,3,4, A(R)=\left(\left\|R^{(1)}\right\|_{\infty} / 4 c\right), B_{i}(R)=\left(\left\|R^{(1)}\right\|_{\infty}\left\|R^{(4)}\right\|_{\infty}+\right.$ $\left.(2 / 3) h_{i}\left\|R^{(3)}\right\|_{\infty}^{2}+2\left\|R^{(2)}\right\|_{\infty}\left\|R^{(3)}\right\|_{\infty}\right) / 384 c, c$ is a constant such that $\min _{0 \leq \rho \leq 1}\left|E_{i}(\rho)\right| \geq c>0, \quad E_{i}(\rho)=\Delta_{i} \rho^{2}+\left(d_{i+1}-d_{i}\right) \rho(1-$ $\rho)+\Delta_{i}(1-\rho)^{2}, \Lambda=\max _{1 \leq i \leq n-1}\left\{2\left(\left|f_{i}\right|+\left|f_{i+1}\right|\right)\right\}, U(\alpha)=$ $\max _{1 \leq i \leq n-1}\left\{U_{i}^{*}(\alpha)+V_{i}^{*}(\alpha) W_{i}^{*}(\alpha)\right\}, U_{i}^{*}(\alpha)=2\left|f_{n}-f_{1}\right|+\left|f_{1}\right|+$ $\left|f_{n}\right|+\left(\left|u_{4, i}+2 \alpha_{i} u_{5, i}\right| /\left|z_{2, i}-z_{1, i}\right|\right), V_{i}^{*}(\alpha)=\left|u_{2, i}\right|+\left|u_{1, i}\right|+\left(\mid u_{3, i} a_{i}+\right.$ $\left.\alpha_{i} u_{4, i}+\alpha_{i}^{2} u_{5, i}|/| z_{2, i}-z_{1, i} \mid\right), W_{i}^{*}(\alpha)=\left(2\left|f_{n}-f_{1}\right| /\left|u_{2, i}-u_{1, i}\right|\right)+$ $\left(\left|d_{1}+d_{n}\right| /\left|z_{2, i}-z_{1, i}\right|\right), u_{1, i}=f_{i}-\alpha_{i} f_{1}, u_{2, i}=f_{i+1}-\alpha_{i} f_{n}, z_{1, i}=$ $a_{i} d_{i}-\alpha_{i} d_{1}, z_{2, i}=a_{i} d_{i+1}-\alpha_{i} d_{n}, u_{3, i}=f_{i} d_{i+1}+f_{i+1} d_{i}, u_{4, i}=$ $-\left(f_{i+1} d_{1}+f_{i} d_{n}+a_{i}\left(f_{n} d_{i}+f_{1} d_{i+1}\right)\right), u_{5, i}=f_{n} d_{1}+f_{1} d_{n}$.

Proof. From (19), the Read-Bajraktarević operator $T^{*}: \mathscr{V}^{*} \times$ $\mathscr{F}^{*} \rightarrow \mathscr{F}^{*}$ such that $(\alpha, g) \mapsto T_{\alpha}^{*} g$ can be rewritten as

$$
\begin{aligned}
T_{\alpha}^{*} g(x) & =F_{i}\left(x, g\left(L_{i}^{-1}(x)\right)\right) \\
& =\alpha_{i} g\left(L_{i}^{-1}(x)\right)+q_{i}\left(L_{i}^{-1}(x), \alpha_{i}\right), \quad x \in I_{i} .
\end{aligned}
$$

From (31), it is clear that the coefficients of the rational quadratic polynomials $q_{i}$ depend continuously on the parameter $\alpha_{i} \geq 0$, and hence we can write $q_{i}(x) \equiv q_{i}\left(x, \alpha_{i}\right)$. If $\alpha=\mathbf{0}$ (zero vector in $\mathscr{V}^{*}$ ), then the classical monotonic quadratic spline $S$ is the fixed point of $T_{0}^{*}$. Let us assume that $r$ is a monotonic rational quadratic FIF associated with a scale vector $\alpha \neq \mathbf{0}$, and $r$ is the fixed point of $T_{\alpha}^{*}$. Since $T_{\alpha}^{*}$ is a contractive operator with the contractive factor $|\alpha|_{\infty}$ (cf. (4)), we have

$$
\left\|T_{\alpha}^{*} r-T_{\alpha}^{*} S\right\|_{\infty} \leq \alpha_{\infty}\|r-S\|_{\infty}
$$

Using (46) and the Mean Value Theorem, we obtain

$$
\begin{aligned}
& \left|T_{\alpha}^{*} S(x)-T_{0}^{*} S(x)\right| \\
& \quad=\left|\alpha_{i} S\left(L_{i}^{-1}(x)\right)+q_{i}\left(L_{i}^{-1}(x), \alpha_{i}\right)-q_{i}\left(L_{i}^{-1}(x), 0\right)\right| \\
& \quad \leq \alpha_{i}\|S\|_{\infty}+\alpha_{i}\left|\frac{\partial q_{i}\left(L_{i}^{-1}(x), \gamma_{i}\right)}{\partial \alpha_{i}}\right|, \quad \gamma_{i}<\alpha_{i} .
\end{aligned}
$$

Now we wish to calculate the bounds of each term in the right-hand side of (48). From Remark 7, the classical monotonic rational quadratic interpolant $S$ is rewritten as

$$
\begin{aligned}
S(x)= & \frac{\Delta_{i} f_{i+1} \rho^{2}}{E_{i}(\rho)}+\frac{f_{i} d_{i+1} \rho(1-\rho)}{E_{i}(\rho)}+\frac{f_{i+1} d_{i} \rho(1-\rho)}{E_{i}(\rho)} \\
& +\frac{\Delta_{i} f_{i}(1-\rho)^{2}}{E_{i}(\rho)} .
\end{aligned}
$$

It is easy to see that for $i=1,2, \ldots, n-1$,

$\operatorname{sgn}\left(E_{i}(\rho)\right)= \begin{cases}+\mathrm{ve} & \text { for monotonically increasing data, } \\ -\mathrm{ve} & \text { for monotonically decreasing data. }\end{cases}$

Using (50), we obtain

$$
\begin{aligned}
\left|E_{i}(\rho)\right| \geq \max & \left\{\left|\Delta_{i}\right| \rho^{2},\left|d_{i}\right| \rho(1-\rho),\right. \\
& \left.\left|d_{i+1}\right| \rho(1-\rho),\left|\Delta_{i}\right|(1-\rho)^{2}\right\} .
\end{aligned}
$$

Using the triangle inequality in (49) and then (51), we have

$$
|S(x)| \leq 2\left(\left|f_{i}\right|+\left|f_{i+1}\right|\right), \quad i=1,2, \ldots, n-1 .
$$

Consequently, the upper bound of $\|S\|_{\infty}$ is given by

$$
\|S\|_{\infty} \leq \Lambda \text {. }
$$

From (31), we rewrite $q_{i}\left(x, \alpha_{i}\right)=U_{i, 2}^{*}\left(\theta, \alpha_{i}\right) / V_{i, 2}^{*}\left(\theta, \alpha_{i}\right)$,

$$
\begin{aligned}
U_{i, 2}^{*}\left(\theta, \alpha_{i}\right)= & \left(f_{i}-\alpha_{i} f_{1}\right) \\
\times & {\left[f_{i+1}-f_{i}-\alpha_{i}\left(f_{n}-f_{1}\right)\right] \theta^{2}+\left(x_{n}-x_{1}\right) } \\
\times & {\left[\left(f_{i} d_{i+1}+f_{i+1} d_{i}\right) a_{i}\right.} \\
& \quad-\alpha_{i}\left\{f_{i+1} d_{1}+f_{i} d_{n}+a_{i}\left(f_{n} d_{i}+f_{1} d_{i+1}\right)\right\} \\
& \left.+\alpha_{i}^{2}\left(f_{n} d_{1}+f_{1} d_{n}\right)\right] \theta(1-\theta) \\
+ & \left(f_{i+1}-\alpha_{i} f_{n}\right)\left[f_{i+1}-f_{i}-\alpha_{i}\left(f_{n}-f_{1}\right)\right] \\
\times & (1-\theta)^{2}, \\
V_{i, 2}^{*}\left(\theta, \alpha_{i}\right)=[ & \left.f_{i+1}-f_{i}-\alpha_{i}\left(f_{n}-f_{1}\right)\right] \theta^{2} \\
+ & \left(x_{n}-x_{1}\right)\left[a_{i}\left(d_{i}+d_{i+1}\right)-\alpha_{i}\left(d_{1}+d_{n}\right)\right] \\
\times & \theta(1-\theta)+\left[f_{i+1}-f_{i}-\alpha_{i}\left(f_{n}-f_{1}\right)\right](1-\theta)^{2} .
\end{aligned}
$$




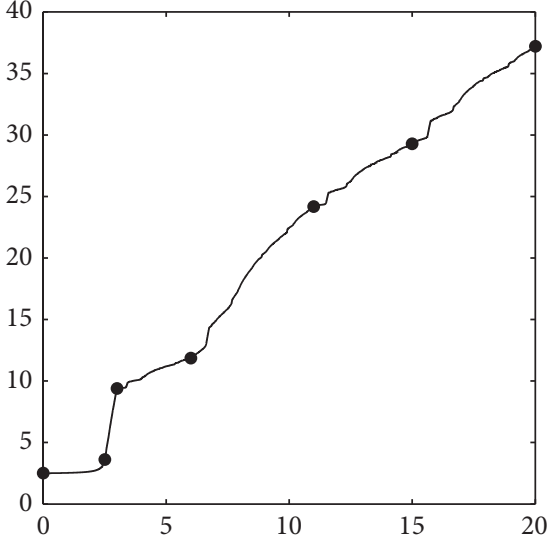

- Interpolation data of $r$

— Increasing rational quadratic FIF $r$

(a) A standard rational quadratic FIF

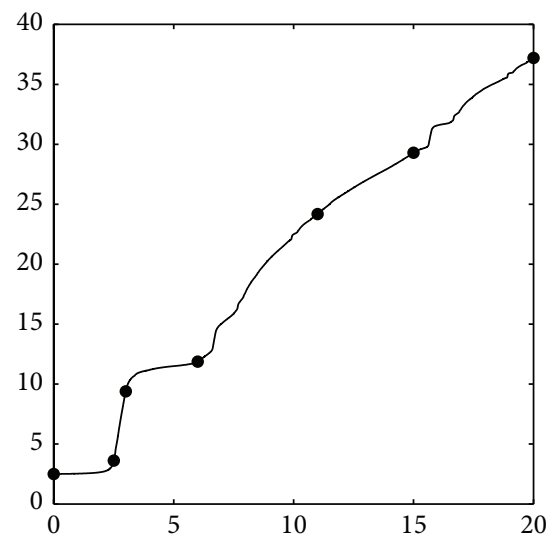

- Interpolation data of $r$

- Increasing rational quadratic FIF $r$ (d) Effects of $\alpha_{3}$ and $\alpha_{5}$ in (a)

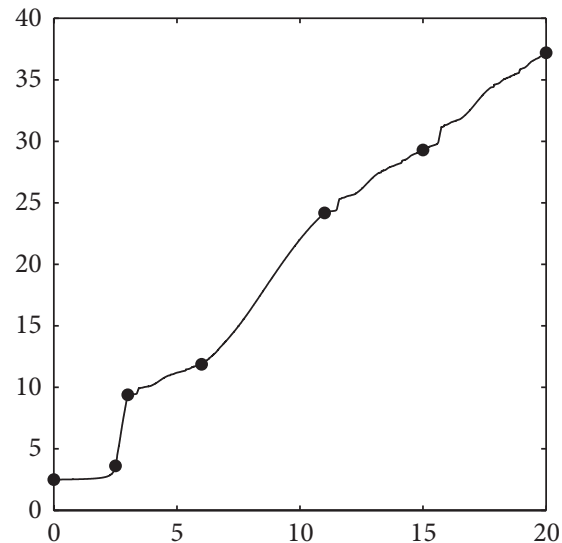

- Interpolation data of $r$

— Increasing rational quadratic FIF $r$

(b) Effects of $\alpha_{4}$ in (a)

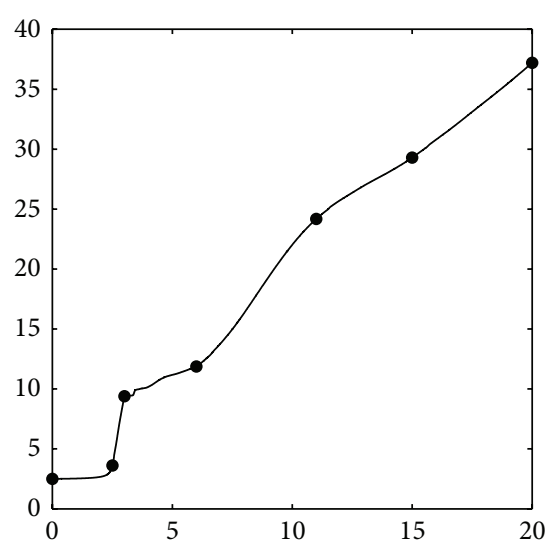

- Interpolation data of $r$ - Increasing rational quadratic FIF $r$

(e) Effects of $\alpha_{4}, \alpha_{5}$, and $\alpha_{6}$ in (a)

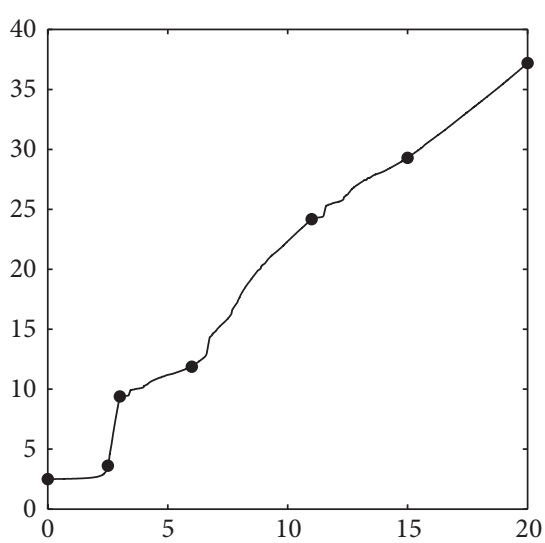

- Interpolation data of $r$

- Increasing rational quadratic FIF $r$

(c) Effects of $\alpha_{6}$ in (a)

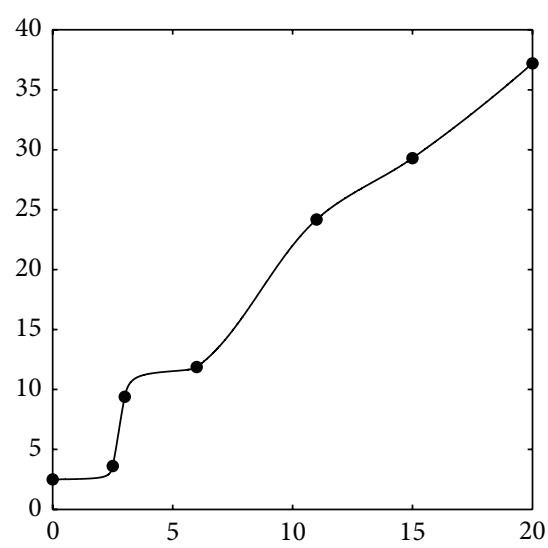

- Interpolation data of $S$ Increasing rational quadratic interpolant $S$

(f) Classical rational quadratic interpolant

FIGURE 4: Monotonicity preserving rational quadratic FIFs.

By differentiating $q_{i}\left(L_{i}^{-1}(x), \alpha_{i}\right)$ partially with respect to $\alpha_{i}$, we get for $i=1,2, \ldots, n-1$, with $\theta=\left(L_{i}^{-1}(x)-x_{1}\right) /\left(x_{n}-x_{1}\right)$, $x \in I_{i}$,

$$
\begin{aligned}
& \frac{\partial q_{i}\left(L_{i}^{-1}(x), \gamma_{i}\right)}{\partial \alpha_{i}} \\
& =\frac{V_{i, 2}^{*}\left(\theta, \alpha_{i}\right)\left(\partial U_{i, 2}^{*}\left(\theta, \alpha_{i}\right) / \partial \alpha_{i}\right)-U_{i, 2}^{*}\left(\theta, \alpha_{i}\right)\left(\partial V_{i, 2}^{*}\left(\theta, \alpha_{i}\right) / \partial \alpha_{i}\right)}{\left(V_{i, 2}^{*}\left(\theta, \alpha_{i}\right)\right)^{2}} .
\end{aligned}
$$

It is easy to verify that

$$
\begin{gathered}
\left|\frac{\partial U_{i, 2}^{*}\left(\theta, \alpha_{i}\right) / \partial \alpha_{i}}{\left|V_{i, 2}^{*}\left(\theta, \alpha_{i}\right)\right|}\right| \leq U_{i}^{*}(\alpha), \quad\left|\frac{U_{i, 2}^{*}\left(\theta, \alpha_{i}\right)}{V_{i, 2}^{*}\left(\theta, \alpha_{i}\right)}\right| \leq V_{i}^{*}(\alpha), \\
\left|\frac{\partial V_{i, 2}^{*}\left(\theta, \alpha_{i}\right) / \partial \alpha_{i}}{\left|V_{i, 2}^{*}\left(\theta, \alpha_{i}\right)\right|}\right| \leq W_{i}^{*}(\alpha) .
\end{gathered}
$$

Using (56) in (55), we get

$$
\begin{array}{r}
\left|\frac{\partial q_{i}\left(L_{i}^{-1}(x), \gamma_{i}\right)}{\partial \alpha_{i}}\right| \leq U_{i}^{*}(\alpha)+V_{i}^{*}(\alpha) W_{i}^{*}(\alpha), \\
i=1,2, \ldots, n-1 .
\end{array}
$$

Consequently, we obtain the following estimation:

$$
\left|\frac{\partial q_{i}\left(L_{i}^{-1}(x), \gamma_{i}\right)}{\partial \alpha_{i}}\right| \leq U(\alpha) .
$$

Substituting (53) and (58) in (48), we obtain for $i=$ $1,2, \ldots, n-1$, and $x \in I$,

$$
\left|T_{\alpha}^{*} S(x)-T_{0}^{*} S(x)\right| \leq \alpha_{i}(\Lambda+U(\alpha)) .
$$

Consequently, we have

$$
\left\|T_{\alpha}^{*} S-T_{0}^{*} S\right\|_{\infty} \leq \alpha_{\infty}(\Lambda+U(\alpha)) .
$$


Combining (47) and (60) with the inequality

$$
\begin{aligned}
\|r-S\|_{\infty} & =\left\|T_{\alpha}^{*} r-T_{0}^{*} S\right\|_{\infty} \\
& \leq\left\|T_{\alpha}^{*} r-T_{\alpha}^{*} S\right\|_{\infty}+\left\|T_{\alpha}^{*} S-T_{0}^{*} S\right\|_{\infty}
\end{aligned}
$$

yields

$$
\|r-S\|_{\infty} \leq \frac{\alpha_{\infty}}{1-\alpha_{\infty}}(\Lambda+U(\alpha))
$$
[32]

Since $R \in \mathscr{C}^{4}(I)$ and $\left|R^{(1)}(x)\right|>0$ on $I$, it is known that

$$
\begin{aligned}
\|S-R\|_{\infty} \leq & h A(R) \max _{1 \leq i \leq n-1}\left\{\left|R_{i}^{(1)}-d_{i}\right|,\left|R_{i+1}^{(1)}-d_{i+1}\right|\right\} \\
& +h^{4} \max _{1 \leq i \leq n-1} B_{i}(R) .
\end{aligned}
$$

Finally, using (62)-(63) with $\|R-r\|_{\infty} \leq\|r-S\|_{\infty}+$ $\|S-R\|_{\infty}$, we get the bound for $\|R-r\|_{\infty}$, and it completes the proof.

Corollary 11. For convergence results of the monotonic rational quadratic FIFs, it is assumed that the estimations of $\max _{1 \leq i \leq n-1}\left\{\left|R_{i}^{(1)}-d_{i}\right|,\left|R_{i+1}^{(1)}-d_{i+1}\right|\right\}, U(\alpha)$, and $\max _{1 \leq i \leq n-1} B_{i}(R)$ are finite for every $h \rightarrow 0$. Note that $\alpha_{\infty}<\max _{1 \leq i \leq n-1} a_{i}=$ $h /\left(x_{n}-x_{1}\right)$; hence one concludes from Theorem 10 that the monotonic rational quadratic FIF converges uniformly to the original function $R$ as $h \rightarrow 0$. Similarly, $O\left(h^{k}\right)(k=2,3,4)$ convergence results can be obtained when the derivative data of $\mathrm{O}\left(h_{i}^{k-1}\right)$ are available with a choice of scaling factors $\alpha_{i}<a_{i}^{k}$ for $i=1,2, \ldots, n-1 ; k=2,3,4$.

\section{Examples}

In order to illustrate the visual quality of the rational quadratic FIFs in fitting of monotonic data, we consider an increasing data set $\{(0,0.5),(2.5,1.61),(3,7.3891),(6,9.8696)$, $(11,22.18),(15,27.3),(20,35.2)\}$ such that $n=7$ in our IFS (37). This data was taken from a function which is differentiable, but its derivative is similar to a continuous and nowhere differentiable function. For the rational quadratic IFS, $L_{i}(x)$ are calculated by using (1). In the evaluation of $F_{i}(x, f)$, the derivative values $d_{i}(i=1,2, \ldots, 7)$ approximated by the Arithmetic Mean Method (cf. Section 3.2) are $d_{1}=0, d_{2}=9.7058, d_{3}=10.0251, d_{4}=1.4401, d_{5}=$ $1.8054, d_{6}=1.4133$, and $d_{7}=1.7467$.

In order to preserve the increasing nature of given increasing data, the restrictions on the scaling factors according to (39) are given by $\alpha_{1}=0, \alpha_{2} \in[0,0.025), \alpha_{3} \in$ $[0,0.0715), \alpha_{4} \in[0,0.2061), \alpha_{5} \in[0,0.1476)$, and $\alpha_{6} \in$ $[0,0.2023)$. A standard rational quadratic FIF (Figure 4(a)) is constructed with a suitable choice of scaling factors (see Table 2). Now, we modify the scaling parameter $\alpha_{4}$ as 0.010 (see Table 2), and the corresponding quadratic FIF is generated (see Figure 4(b)). In comparison with Figure 4(a), we observe that there are visually pleasing changes in the

\begin{tabular}{|c|c|}
\hline Figure no. & Scaling factors \\
\hline Figure 4(a) & $\begin{array}{c}\alpha_{1}=0, \alpha_{2}=0.021, \alpha_{3}=0.07, \alpha_{4}=0.202 \\
\alpha_{5}=0.142, \alpha_{6}=0.201\end{array}$ \\
\hline Figure 4(b) & $\begin{array}{c}\alpha_{1}=0, \alpha_{2}=0.021, \alpha_{3}=0.07, \alpha_{4}=0.010 \\
\alpha_{5}=0.142, \alpha_{6}=0.201\end{array}$ \\
\hline Figure 4(c) & $\begin{array}{c}\alpha_{1}=0, \alpha_{2}=0.021, \alpha_{3}=0.07, \alpha_{4}=0.202 \\
\alpha_{5}=0.142, \alpha_{6}=0.010\end{array}$ \\
\hline Figure 4(d) & $\begin{array}{c}\alpha_{1}=0, \alpha_{2}=0.021, \alpha_{3}=0.010, \alpha_{4}=0.202 \\
\alpha_{5}=0.010, \alpha_{6}=0.201\end{array}$ \\
\hline Figure 4(e) & $\begin{array}{c}\alpha_{1}=0, \alpha_{2}=0.021, \alpha_{3}=0.07, \alpha_{4}=0.010 \\
\alpha_{5}=0.010, \alpha_{6}=0.010\end{array}$ \\
\hline Figure 4(f) & $\alpha_{1}=\alpha_{2}=\alpha_{3}=\alpha_{4}=\alpha_{5}=\alpha_{6}=0$ \\
\hline
\end{tabular}
graph of the rational quadratic FIF in the fourth sub-interval $\left[x_{4}, x_{5}\right]$ of Figure $4(\mathrm{~b})$, and the changes in other intervals are
TABLE 2: Scaling factors used in the construction of the rational quadratic FIFs.

negligible. Similarly, we modify $\alpha_{6}$ (see Table 2 ) with respect to Figure 4(a), and the corresponding rational quadratic FIF is generated in Figure 4(c). In comparison with Figure 4(a), it is observed that the graph of the rational quadratic FIF in the sixth sub-interval $\left[x_{6}, x_{7}\right]$ converging to the straight line between $\left(x_{6}, f_{6}\right)$ and $\left(x_{7}, f_{7}\right)$ as $\alpha_{6} \rightarrow 0^{+}$and variations in the other sub-intervals are not noticeable. Similarly we modify $\alpha_{3}$ and $\alpha_{5}$ (see Table 2 ) with respect to Figure 4(a), and the appropriate rational quadratic FIF is constructed in Figure 4(d). By analyzing Figure 4(d) with respect to Figure 4(a), the perceptible effects are observed in the third and fifth sub-intervals. In particular, the graph of the rational quadratic FIF in the third sub-interval $\left[x_{3}, x_{4}\right]$ is converging to a concave shape as $\alpha_{3} \rightarrow 0^{+}$. Again a simultaneous modification of $\alpha_{4}, \alpha_{5}$, and $\alpha_{6}$ (see Table 2) yields the rational quadratic FIF in Figure 4(e), and we observe that the individual effects of $\alpha_{4}, \alpha_{6}$, and $\alpha_{5}$, respectively, from Figures 4(b)-4(d) are reflected in Figure 4(e). From the above discussion, it is interesting to see that although the rational quadratic FIF $r$ is a global interpolant, the effects of the scaling factors $\alpha_{3}, \alpha_{4}, \alpha_{5}$, and $\alpha_{6}$ are very much local in nature (see Figures 4(a)-4(e)). Using the zero scaling vector, we generate the classical rational quadratic interpolant in Figure 4(f). From the above demonstration, we conclude that proper interactive adjustments of the scaling factors give us a wide variety of monotonic fractal curves that can be used in various scientific and engineering problems for aesthetic modifications or where the classical rational quadratic interpolant is insufficient and unsatisfactory. From (40), it easy to see that the scaling vector for the rational fractal function $r^{\prime}$ is

$$
\alpha^{*}=\left(\alpha_{1}^{*}, \alpha_{2}^{*}, \ldots, \alpha_{n-1}^{*}\right)=\left(\frac{\alpha_{1}}{a_{1}}, \frac{\alpha_{2}}{a_{2}}, \ldots, \frac{\alpha_{n-1}}{a_{n-1}}\right) .
$$

Again from (18) and (40), it easy to verify that $r^{\prime}$ interpolatespagebreak the data $\left\{\left(x_{i}, d_{i}\right): i=1,2, \ldots, n\right\}$. In this example, the interpolation data set for $r^{\prime}$ is $\{(0,0),(2.5,9.7058),(3,10.0251),(6,1.4401),(11,1.8054)$, $(15,1.4133),(20,1.7467)\}$.

The derivative functions of the rational quadratic FIFs in Figures 4(a)-4(e) are constructed in Figures 5(a)-5(e), respectively. The rational fractal functions in 


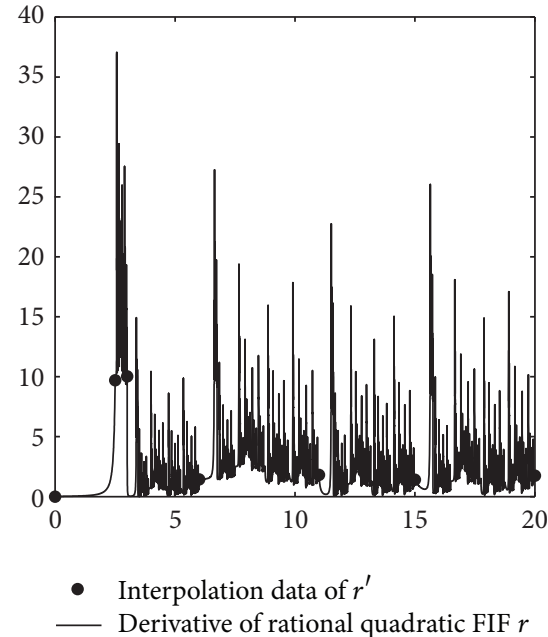

(a) Derivative of rational quadratics FIF in Figure 4(a)

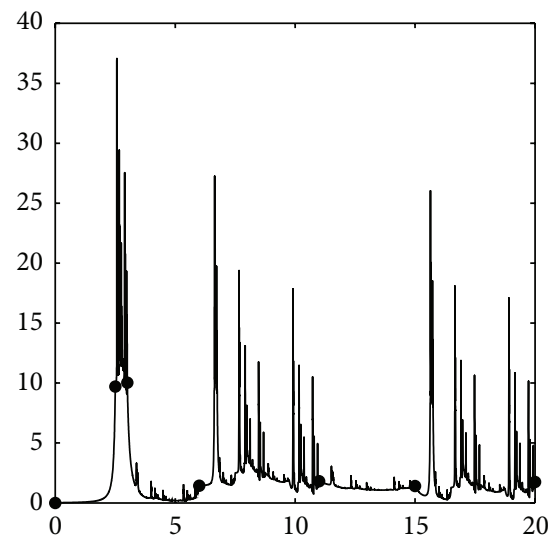

- Interpolation data of $r^{\prime}$ — Derivative of rational quadratic FIF $r$

(d) Derivative of rational quadratics FIF in Figure 4(d)

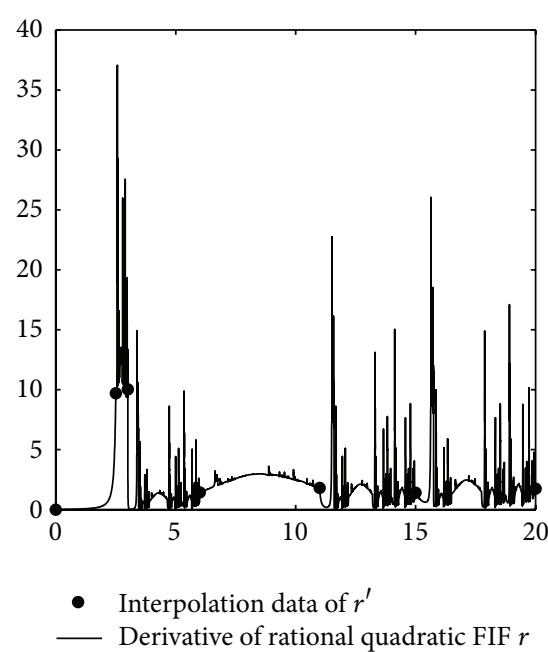

(b) Derivative of rational quadratics FIF in Figure 4(b)

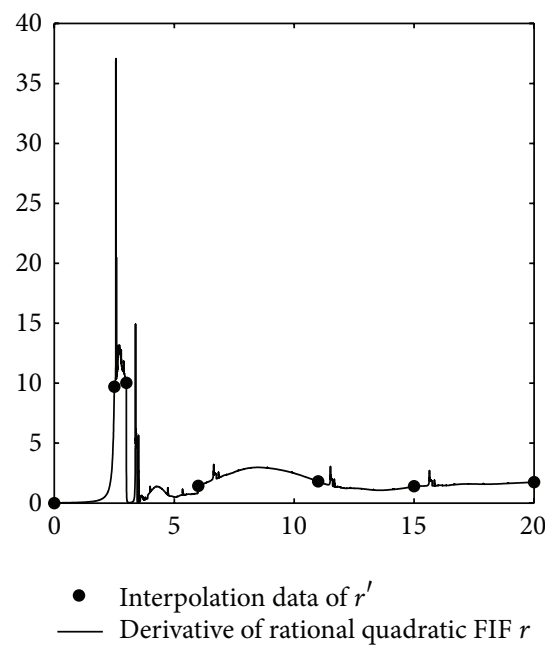

(e) Derivative of rational quadratics FIF in Figure 4(e)

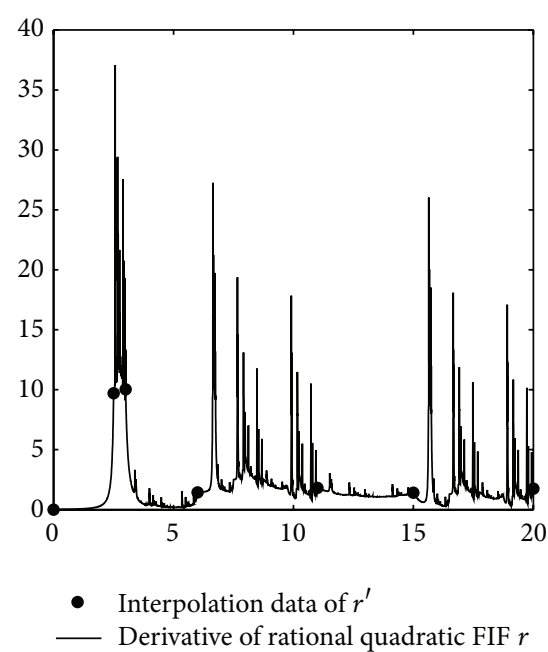

(c) Derivative of rational quadratics FIF in Figure 4(c)

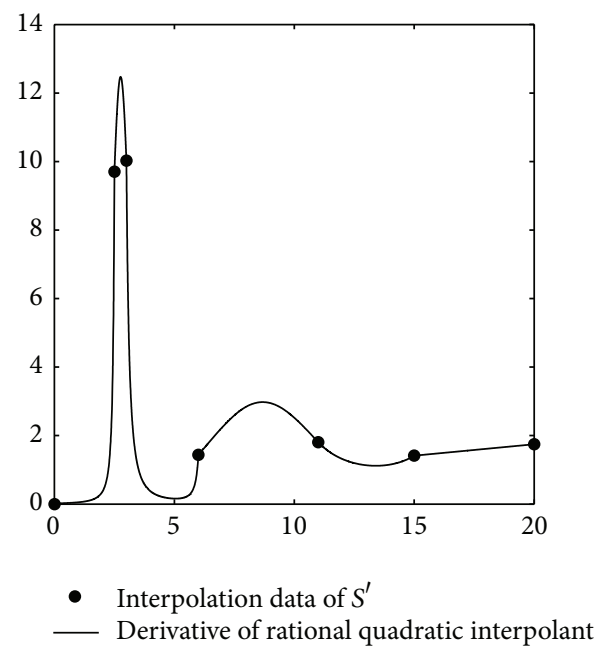

(f) Derivative of classical rational quadratic interpolant in Figure 4(f)

FIgURE 5: Derivatives of the rational quadratic FIFs and the classical rational quadratic interpolant.

Figures 5(a)-5(e) are typical fractal functions close to continuous but nowhere differentiable function. By taking the rational quadratic FIF in Figure 4(a) as the original function, we have calculated the uniform distance between this original function and the rational quadratic FIFs in Figures 4(b) and 4(f) (see Table 3). We observe that deviations in the rational quadratic FIF in Figure 4(a) due to $\alpha_{4}$ are more than that of the scaling factor $\alpha_{6}$.

Also we have calculated the uniform distance between their derivatives (see Table 3 ). The effects of the scaling factors $\alpha_{4}$ and $\alpha_{6}$ are very much prominent in the fourth and sixth subintervals of Figures 4(b)-4(c), respectively, in comparison with Figure 4(a), but they render major effects in their derivatives (see Figures 5(b)-5(c) and Table 3).

The rational FIFs in Figures 5(a)-5(e) are irregular in nature over the interval $\left[x_{1}, x_{7}\right]$, whereas the derivative of
TABLE 3: Uniform distances between rational quadratic FIFs and their derivatives.

\begin{tabular}{lccc}
\hline $\begin{array}{l}\text { Rational } \\
\text { quadratic FIF }\end{array}$ & $\begin{array}{c}\text { Uniform } \\
\text { distance with } \\
\text { Figure 4(a) }\end{array}$ & $\begin{array}{c}\text { Derivative of } \\
\text { rational } \\
\text { quadratic FIF }\end{array}$ & $\begin{array}{c}\text { Uniform } \\
\text { distance with } \\
\text { Figure 5(a) }\end{array}$ \\
\hline Figure 4(b) & 1.4795 & Figure 5(b) & 2.3241 \\
Figure 4(c) & 0.9089 & Figure 5(c) & 2.7532 \\
Figure 4(d) & 0.7003 & Figure 5(d) & 1.0213 \\
Figure 4(e) & 0.4393 & Figure 5(e) & 1.4934 \\
Figure 4(f) & 1.9801 & Figure 5(f) & 3.0612 \\
\hline
\end{tabular}

the classical interpolant is a piecewise smooth in the interval $\left[x_{1}, x_{7}\right]$ (see Figure 5(f)). Because of this reason, if the original function is $C^{1}$-smooth and monotonic but its derivative is a continuous nowhere differentiable function, then our 
rational quadratic FIF $r$ is an ideal tool to approximate such function instead of the classical rational quadratic interpolant, whose derivative is a piece-wise smooth function. As the approximation of an original function like Figure 4(a) and its derivative is concerned, numerical data given in Table 3 reveals that proposed rational quadratic FIF $r$ is a better approximant over the classical rational quadratic interpolant $S$.

\section{Conclusion}

In the present paper, the notion of rational fractal interpolation without shape parameter is introduced in the literature for the first time. In particular, the $\mathscr{C}^{1}$-rational quadratic FIFs that contain parameters, namely, the scaling factors, are developed for representation of a given real data set. The derivative values are approximated at grids by the Arithmetic Mean Method and Geometric Mean Method so that the $\mathscr{C}^{1}$-smoothness of quadratic FIF is maintained. A rational quadratic FIF need not be monotonic for arbitrary choice of scaling factors for given monotonic data in general. To preserve the monotonicity feature of a data set, the sufficient conditions are prescribed through the scaling factors on the associated IFS. Therefore, the data dependent scaling factors are introduced in this work for shape preserving rational FIFs. A uniform error bound is deduced between the monotonic rational quadratic FIF and an original function. From this, it is possible to achieve $O\left(h^{k}\right)(k=1,2,3,4)$ convergence, when accurate derivative values of order $O\left(h_{i}^{k-1}\right)$ are available at grids, and the scaling factors are taken as $0 \leq \alpha_{i}<a_{i}^{k}$ for $i=1,2, \ldots, n-1$. The role of scaling factors in local control of shape of rational quadratic FIFs is demonstrated through suitable examples. The effects of scaling factors in the derivative of rational quadratic FIF may be local, moderately local, or global, and it varies according to the interpolation data. The proper choice of scaling factors, for which the uniform errors $\|r-R\|_{\infty}$ and $\left\|r^{\prime}-R^{\prime}\right\|_{\infty}$ are minimum, is an open problem in the FIF optimization technique, where the original function $R \in \mathscr{C}^{1}$ is such that its derivative $R^{\prime}$ is similar to a continuous no-where-differentiable function. Since the construction of a rational FIF depends on the choice of scaling factors, one can construct different shapes of monotonicity preserving $\mathscr{C}^{1}$-rational quadratic FIFs for the same data by varying its scale vector. Due to this added flexibility, our rational quadratic FIFs will have huge number of applications in computer visualization, computational geometry, object recognition, shape abstraction and modeling, CAD, reverse engineering, and other scientific applications as compared to the classical rational quadratic splines.

\section{Conflict of Interests}

The authors declare that they have no competing interests.

\section{Acknowledgment}

The partial support of the Department of Science and Technology of Government of India (SERC DST Project no. SR/S4/MS: 694/10) is gratefully acknowledged.

\section{References}

[1] M. F. Barnsley, "Fractal functions and interpolation," Constructive Approximation, vol. 2, no. 4, pp. 303-329, 1986.

[2] J. E. Hutchinson, "Fractals and self-similarity," Indiana University Mathematics Journal, vol. 30, no. 5, pp. 713-747, 1981.

[3] M. A. Navascués and M. V. Sebastian, "Fitting curves by fractal interpolation: an application to the quantification of cognitive brain processes," in Thinking in Patterns: Fractals and Related Phenomena in Nature, M. M. Novak, Ed., pp. 143-154, World Scientific, River Edge, NJ, USA, 2004.

[4] M. A. Navascués and A. K. B. Chand, "Fundamental sets of fractal functions," Acta Applicandae Mathematicae, vol. 100, no. 3, pp. 247-261, 2008.

[5] A. K. B. Chand and M. A. Navascués, "Generalized hermite fractal interpolation," Revista de la Real Academia de Ciencias Exactas, Físicas, Químicas y Naturales de Zaragoza, Series 2, vol. 64, pp. 107-120, 2009.

[6] M. F. Barnsley and A. N. Harrington, "The calculus of fractal interpolation functions," Journal of Approximation Theory, vol. 57, no. 1, pp. 14-34, 1989.

[7] A. K. B. Chand and G. P. Kapoor, "Generalized cubic spline fractal interpolation functions," SIAM Journal on Numerical Analysis, vol. 44, no. 2, pp. 655-676, 2006.

[8] A. K. B. Chand and G. P. Kapoor, "Spline coalescence hidden variable fractal interpolation functions," Journal of Applied Mathematics, vol. 2006, Article ID 36829, 17 pages, 2006.

[9] A. K. B. Chand and P. Viswanathan, "Cubic hermite and cubic spline fractal interpolation functions," AIP Conference Proceedings, vol. 1479, pp. 1467-1470, 2012.

[10] A. K. B. Chand and P. Viswanathan, "A constructive approach to cubic hermite fractal interpolation function and its constrained aspects," BIT Numerical Mathematics, vol. 53, no. 4, pp. 841-865, 2013.

[11] M. A. Navascués and M. V. Sebastián, "Smooth fractal interpolation," Journal of Inequalities and Applications, vol. 2006, Article ID 78734, 20 pages, 2006.

[12] W. C. Strahle, "Turbulent combustion data analysis using fractals," AIAA Journal, vol. 29, no. 3, pp. 409-417, 1991.

[13] D. S. Mazel and M. H. Hayes, "Using iterated function systems to model discrete sequences," IEEE Transactions on Signal Processing, vol. 40, no. 7, pp. 1724-1734, 1992.

[14] L. I. Levkovich-Maslyuk, "Wavelet-based determination of generating matrices for fractal interpolation functions," Regular \& Chaotic Dynamics, vol. 3, no. 2, pp. 20-29, 1998.

[15] K. Berkner, "A wavelet based solution to the inverse problem for fractal interpolation functions," in Fractals in Engineering, J. L. Véhel, E. Lutton, and C. Tricot, Eds., pp. 81-92, Springer, London, UK, 1997.

[16] E. Guérin, E. Tosan, and A. Baskurt, "A fractal approximation of curves," Fractals, vol. 9, no. 1, pp. 95-103, 2001.

[17] I. J. Schoenberg, "Contributions to the problem of approximation of equidistant data by analytic functions, part a:on the problem of smoothing of graduation, a first class of analytic 
approximation," Quarterly of Applied Mathematics, vol. 4, pp. 45-88, 1946.

[18] I. J. Schoenberg, "Contributions to the problem of approximation of equidistant data by analytic functions. part b: on the problem of osculatory formulae," Quarterly of Applied Mathematics, vol. 4, pp. 112-141, 1946.

[19] D. G. Schweikert, "An interpolation curve using a spline in tension," Journal of Mathematical Physics, vol. 45, pp. 312-317, 1966.

[20] H. Späth, Spline Algorithms for Curves and Surfaces, Utilitas Mathematica, Winnipeg, Canada, 1974.

[21] G. M. Nielson, "Some piecewise polynomial alternatives to splines under tension," in Computer-Aided Geometric Design, R. E. Barnhill and R. F. Riesenfeld, Eds., pp. 209-235, Academic Press, New York, NY, USA, 1974.

[22] C. de Boor, A Practical Guide to Splines, vol. 27 of Applied Mathematical Sciences, Springer, New York, NY, USA, 1978.

[23] S. Pruess, "Alternatives to the exponential spline in tension," Mathematics of Computation, vol. 33, no. 148, pp. 1273-1281, 1979.

[24] F. N. Fritsch and R. E. Carlson, "Monotone piecewise cubic interpolation," SIAM Journal on Numerical Analysis, vol. 17, no. 2, pp. 238-246, 1980.

[25] F. N. Fritsch and J. Butland, "A method for constructing local monotone piecewise cubic interpolants," SIAM Journal on Scientific and Statistical Computing, vol. 5, no. 2, pp. 300-304, 1984.

[26] P. Costantini, "On monotone and convex spline interpolation," Mathematics of Computation, vol. 46, no. 173, pp. 203-214, 1986.

[27] P. Costantini, "Co-monotone interpolating splines of arbitrary degree-a local approach," SIAM Journal on Scientific and Statistical Computing, vol. 8, no. 6, pp. 1026-1034, 1987.

[28] P. Costantini and M. Rossana, "Monotone and convex cubic spline interpolation," Calcolo, vol. 21, no. 3, pp. 281-294, 1984.

[29] D. F. McAllister and J. A. Roulier, "An algorithm for computing a shape-preserving osculatory quadratic spline," ACM Transactions on Mathematical Software, vol. 7, no. 3, pp. 331-347, 1981.

[30] L. L. Schumaker, "On shape preserving quadratic spline interpolation," SIAM Journal on Numerical Analysis, vol. 20, no. 4, pp. 854-864, 1983.

[31] A. Lahtinen, "On the choice of the initial value of a quadratic spline in positive interpolation," Journal of Computational and Applied Mathematics, vol. 69, no. 1, pp. 13-25, 1996.

[32] J. A. Gregory and R. Delbourgo, "Piecewise rational quadratic interpolation to monotonic data," IMA Journal of Numerical Analysis, vol. 2, no. 2, pp. 123-130, 1982.

[33] J. W. Schmidt and W. Heß, "Positive interpolation with rational quadratic splines," Computing, vol. 38, no. 3, pp. 261-267, 1987.

[34] J. W. Schmidt, W. Heß, and Th. Nordheim, "Shape preserving histopolation using rational quadratic splines," Computing, vol. 44, no. 3, pp. 245-258, 1990.

[35] M. P. Groover and E. W. Zimmers, CAD/CAM: Computer-Aided Design and Manufacturing, Pearson Education, Upper Saddle River, NJ, USA, 1997.

[36] G. Farin, Curves and Surfaces for Computer Aided Geometric Design: A Practical Guide, Computer Science and Scientific Computing, Academic Press, San Diego, Calif, USA, 1990.

[37] M. Sakai and J. W. Schmidt, "Positive interpolation with rational splines," BIT Numerical Mathematics, vol. 29, no. 1, pp. 140-147, 1989.
[38] J. A. Gregory, M. Sarfraz, and P. K. Yuen, "Interactive curve design using $\mathscr{C}^{2}$ rational splines," Computers and Graphics, vol. 18, no. 2, pp. 153-159, 1994.

[39] E. H. Kaufman Jr. and G. D. Taylor, "Approximation and interpolation by convexity-preserving rational splines," Constructive Approximation, vol. 10, no. 2, pp. 275-283, 1994.

[40] K. W. Brodlie and S. Butt, "Preserving convexity using piecewise cubic interpolation," Computers and Graphics, vol. 15, no. 1, pp. $15-23,1991$.

[41] Y. Fisher, Fractal Image Compression: Theory and Application, Springer, New York, NY, USA, 1995.

[42] J. A. Gregory and R. Delbourgo, "The determination of derivative parameters for a monotonic rational quadratic interpolant," IMA Journal of Numerical Analysis, vol. 5, no. 4, pp. 397-406, 1985. 


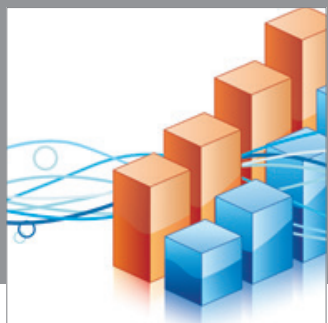

Advances in

Operations Research

mansans

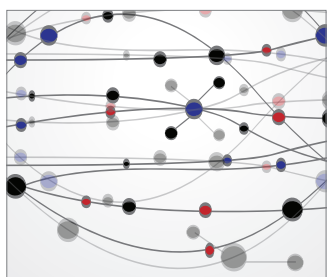

The Scientific World Journal
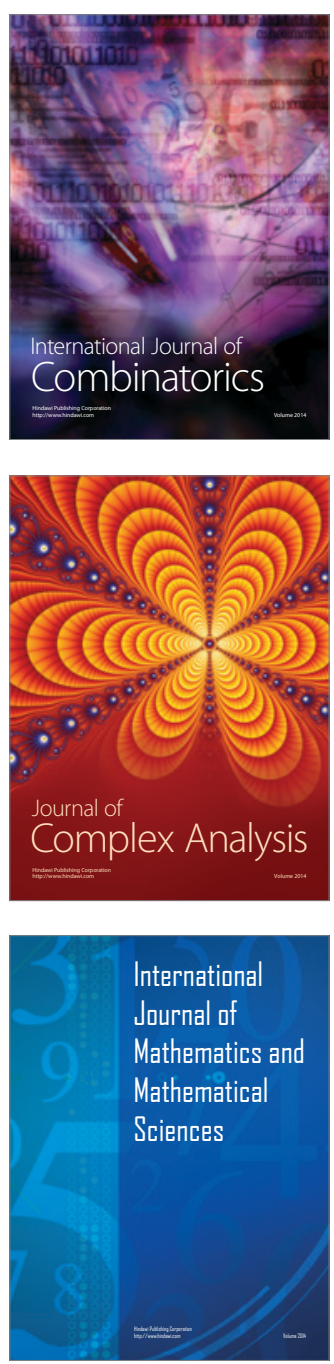
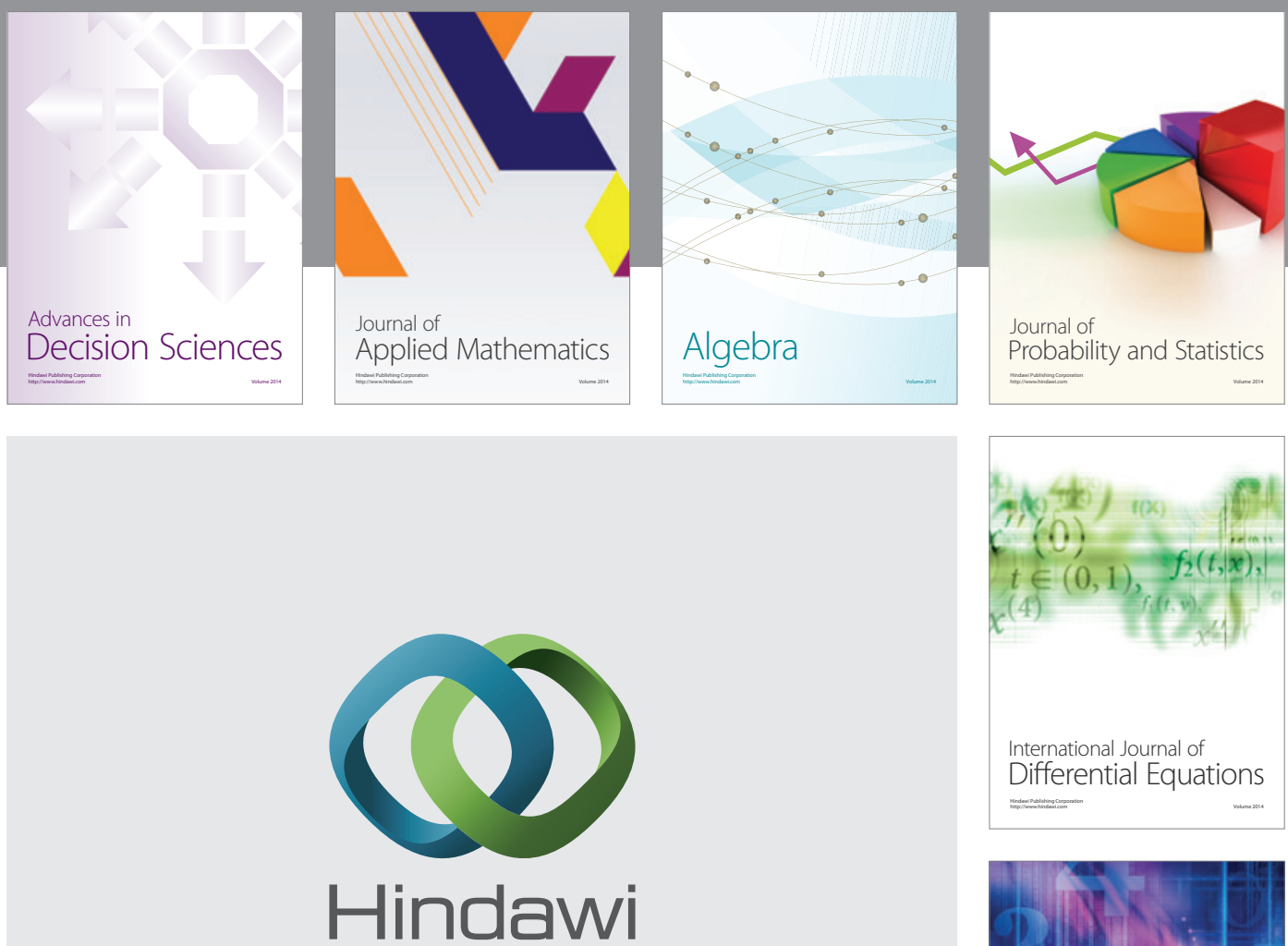

Submit your manuscripts at http://www.hindawi.com
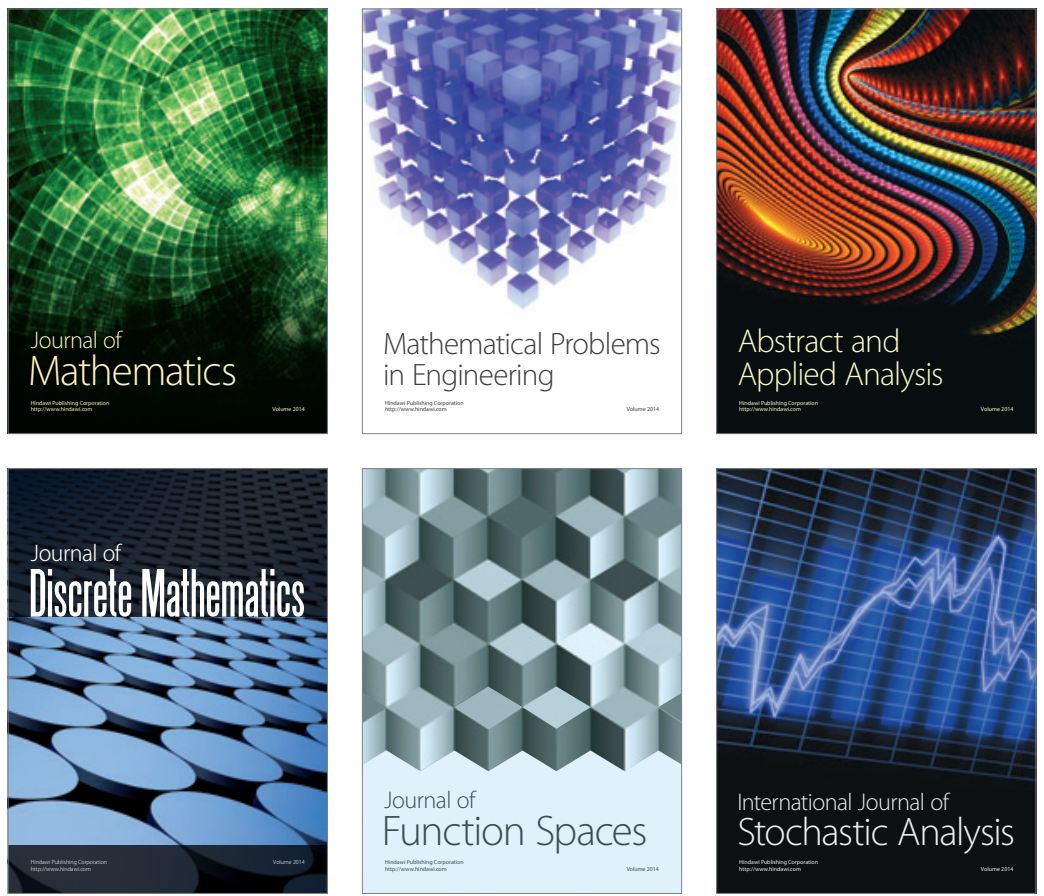

Journal of

Function Spaces

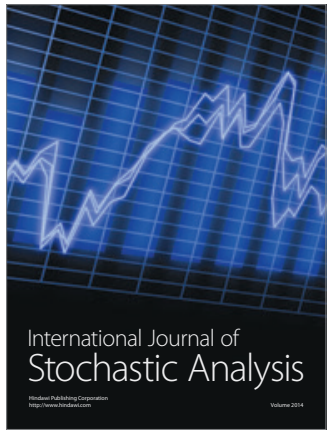

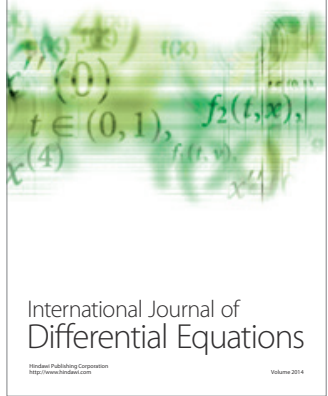
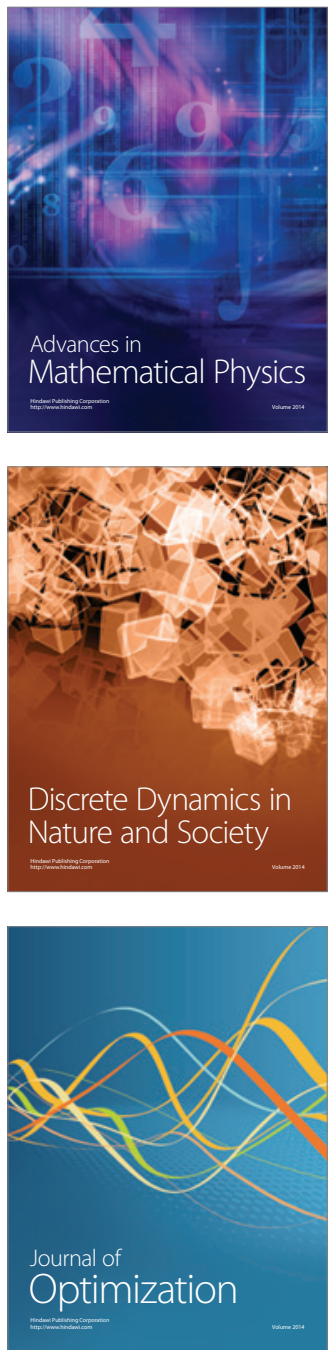